\title{
Search for Evidence of the Type-III Seesaw Mechanism in Multilepton Final States in Proton-Proton Collisions at $\sqrt{s}=13 \mathrm{TeV}$
}

\author{
A. M. Sirunyan et al. \\ (CMS Collaboration) \\ (Received 26 August 2017; published 1 December 2017)
}

\begin{abstract}
A search for a signal consistent with the type-III seesaw mechanism in events with three or more electrons or muons is presented. The data sample consists of proton-proton collisions at $\sqrt{s}=13 \mathrm{TeV}$ collected by the CMS experiment at the LHC in 2016 and corresponds to an integrated luminosity of $35.9 \mathrm{fb}^{-1}$. Selection criteria based on the number of leptons and the invariant mass of oppositely charged lepton pairs are used to distinguish the signal from the standard model background. The observations are consistent with the expectations from standard model processes. The results are used to place limits on the production of heavy fermions of the type-III seesaw model as a function of the branching ratio to each lepton flavor. In the scenario of equal branching fractions to each lepton flavor, heavy fermions with masses below $840 \mathrm{GeV}$ are excluded. This is the most sensitive probe to date of the type-III seesaw mechanism.
\end{abstract}

DOI: 10.1103/PhysRevLett.119.221802

The discovery of neutrino oscillations established that neutrinos have mass [1]. Among proposed extensions of the standard model (SM) that explain neutrino mass, models based on the seesaw mechanism are an appealing possibility [2-10]. The seesaw mechanism introduces new heavy particles that couple to leptons and to a Higgs boson $(H)$ doublet, and accounts for the smallness of neutrino masses [11-13]. Within the type-III seesaw model [8], the neutrino is considered a Majorana particle whose mass arises from the mediation of new massive fermions that form an SU(2) triplet of heavy Dirac charged leptons $\Sigma^{ \pm}$and a heavy Majorana neutral lepton $\Sigma^{0}$. In proton-proton $(p p)$ collisions, these massive fermions may be pair-produced through leading order electroweak interactions, in both charged-charged and charged-neutral pairs. The renewed interest in the type-III seesaw models [14-19] emphasizes the importance of exploring such signatures at the CERN LHC.

We conduct a search for these new massive fermions by examining final states with at least three electrons and muons (including those that come from the decays of tau leptons) [20,21]. Such final states arise from these decays: $\Sigma^{ \pm} \rightarrow W^{ \pm} \nu_{\ell}, \quad \Sigma^{ \pm} \rightarrow Z \ell^{ \pm}, \quad \Sigma^{ \pm} \rightarrow \mathrm{H} \ell^{ \pm}, \quad \Sigma^{0} \rightarrow W^{ \pm} \ell^{\mp}$, $\Sigma^{0} \rightarrow Z \nu_{\ell}$, and $\Sigma^{0} \rightarrow \mathrm{H} \nu_{\ell}$, where $\ell=e, \mu$, or $\tau$. The branching fractions are mass dependent. A complete decay chain example would be $\Sigma^{ \pm} \Sigma^{0} \rightarrow W^{ \pm} \nu W^{ \pm} \ell^{\mp} \rightarrow$ $\ell^{ \pm} \nu \nu \ell^{ \pm} \nu \ell^{\mp}$. In this search, we consider all 27 different

*Full author list given at the end of the article.

Published by the American Physical Society under the terms of the Creative Commons Attribution 4.0 International license. Further distribution of this work must maintain attribution to the author(s) and the published article's title, journal citation, and DOI. production and decay processes resulting from the decays of each of the $\Sigma^{0} \Sigma^{+}, \Sigma^{0} \Sigma^{-}$, and $\Sigma^{+} \Sigma^{-}$pairs to the nine different pairs of $W, Z$, and $H$ bosons.

In the model used in this analysis [22], the $\Sigma^{ \pm}$and $\Sigma^{0}$ decays are prompt, and gauge invariance ensures that they are degenerate in mass at tree level. The branching fraction $\left(B_{\ell}\right)$ of a heavy fermion to a lepton of flavor $\ell$ is proportional to $\left|V_{\ell}\right|^{2} /\left(\left|V_{e}\right|^{2}+\left|V_{\mu}\right|^{2}+\left|V_{\tau}\right|^{2}\right)$, where $V_{\ell}$ is the heavy-light fermion mixing angle. A prior CMS search using $7 \mathrm{TeV}$ data excluded heavy fermions with mass $\left(m_{\Sigma}\right)$ below $179 \mathrm{GeV}$ [23], probing a scenario where the branching fractions to all lepton flavors are equal (flavor-democratic scenario, $B_{e}=B_{\mu}=B_{\tau}$ ). Using $8 \mathrm{TeV}$ data, the ATLAS Collaboration similarly excluded $m_{\Sigma}$ below $335 \mathrm{GeV}$, assuming that the $\Sigma$ 's decay only to the first two generations of leptons [24]. In this Letter, we probe all values of $B_{\ell}$ for each flavor of $\ell$, subject to the constraint $B_{e}+B_{\mu}+B_{\tau}=1$.

We pursue a search in final states with at least three charged leptons. The backgrounds can be classified as the irreducible ones due to the production and decay of dibosons ( $W Z$ and $Z Z$ production), and the reducible ones due to the leptonic decay of $Z+$ jets or $t \bar{t}+$ jets accompanied by leptons originating from heavy quark decay, or from misidentification of jets as leptons. In addition, small irreducible contributions to the background arise from other SM processes such as $t \bar{t} W, t \bar{t} Z$, triboson $(Z Z Z, W W Z$, etc.), and Higgs boson production.

The central feature of the CMS apparatus is a superconducting solenoid of $6 \mathrm{~m}$ internal diameter, providing a magnetic field of $3.8 \mathrm{~T}$. Within the solenoid volume are a silicon pixel and strip tracker, a lead tungstate crystal electromagnetic calorimeter (ECAL), and a brass and scintillator hadron calorimeter, each composed of a barrel and two end cap sections. Forward calorimeters extend the 
pseudorapidity $(\eta)$ coverage provided by the barrel and end cap detectors. Muons are detected in gas-ionization chambers embedded in the steel flux-return yoke outside the solenoid. A detailed description of the CMS detector can be found in Ref. [25].

The data sample consists of $p p$ collisions at $\sqrt{s}=$ $13 \mathrm{TeV}$ collected by the CMS experiment at the LHC in 2016, corresponding to an integrated luminosity of $35.9 \mathrm{fb}^{-1}$. The data for this search are collected using several dilepton triggers based on a two-level trigger system [26]. The double-electron (double-muon) trigger requires two loosely isolated electrons (muons). The transverse momentum $\left(p_{T}\right)$ thresholds on the leading (subleading) lepton are 23 (12) GeV for the double electron, and 17 (8) $\mathrm{GeV}$ for the double-muon trigger, respectively. We also use two muon-electron cross-trigger combinations that require a $p_{T}>23 \mathrm{GeV}$ loosely isolated muon (electron) and a $p_{T}>8 \mathrm{GeV}$ loosely isolated electron (muon).

The signal is simulated using MADGrAPH5_aMC@NLO v2.2.2 [27] at leading order precision using the NNPDF30_ lo_as_0130_nf_4 [28] parton distribution functions (PDFs). The signal cross section is calculated at next-to-leading order (NLO) plus next-to-leading logarithmic accuracy, assuming that the heavy leptons, $\Sigma^{ \pm, 0}$, are $\mathrm{SU}(2)$ triplet fermions $[29,30]$. The diboson backgrounds are generated at NLO with POWHEG 2.0 [31-33]. Backgrounds from Higgs boson production are generated using the POWHEG and JHUGEN 6.2.8 [34-37] generators. Triboson backgrounds are generated using MADGRAPH5_aMC@NLO. Bosonic decays, parton showering, fragmentation, and hadronization are simulated for all samples using PYTHIA 8.212 [38,39].

The response of the CMS detector is simulated using dedicated software based on the GEANT4 toolkit [40]. The presence of multiple $p p$ interactions in the same or adjacent bunch crossing (pileup) [41] is incorporated by simulating additional interactions that are both in time and out of time with the collision.

Events satisfying the trigger are required to pass additional off-line selections. Electrons and muons must satisfy $p_{T}>10 \mathrm{GeV}$ and $|\eta|<2.5$ ( $|\eta|<2.4$ for muons). Electrons are reconstructed using Gaussian sum filter tracks matched to ECAL superclusters [42]. Muons are reconstructed using the particle-flow (PF) algorithm [43] that utilizes measurements from the tracker, calorimeter, and muon systems to reconstruct candidate muons, photons, and hadrons. Tracks of candidate electrons and muons must satisfy quality requirements and should match geometrically with either energy deposits in the ECAL (for electrons) or tracks in the muon detectors (for muons).

Jets are reconstructed from the PF candidates and electrons using the anti- $k_{T}$ algorithm [44] with distance parameter $R=0.4$. Jets are required to have $p_{T} \geq 30 \mathrm{GeV}$ and $|\eta| \leq 3.0$ and satisfy quality criteria that remove contributions from pileup. The missing transverse momentum vector $\vec{p}_{T}^{\text {miss }}$ is defined as the projection onto the plane perpendicular to the beam axis of the negative vector sum of the momenta of all reconstructed PF objects in an event [45]. Its magnitude is referred to as $p_{T}^{\text {miss }}$. Jet energy corrections are applied to all jets and also propagated to the calculation of $\vec{p}_{T}^{\text {miss }}$.

The reducible backgrounds include leptons inside or near jets and leptons from heavy quark decays. In addition, hadrons that reach the muon detectors, or hadronic showers with large electromagnetic energy fractions can be misidentified as leptons. Henceforth, we refer to all leptons not arising from vector boson decay (either directly or via an intermediate tau lepton) as misidentified leptons. Backgrounds from misidentified leptons are significantly reduced by an isolation requirement comparing the $p_{T}$ of a lepton to the summed $p_{T}$ of particles in its neighborhood. We utilize relative isolation, which is defined as the scalar $p_{T}$ sum, normalized to the lepton $p_{T}$, of photon and hadron PF candidates within a cone around the lepton. This relative isolation is required to be less than $25 \%$ within a cone of size $R=\sqrt{(\Delta \eta)^{2}+(\Delta \phi)^{2}}=0.4$ for muons, where $\phi$ is azimuthal angle in radians. For electrons, the relative isolation within a cone of $R=0.3$ must be less than $7 \%(8 \%)$ for the reconstructed energy deposits situated in the ECAL barrel $[|\eta|<1.479]$ (ECAL end cap $[|\eta|>1.479])$. Charged tracks returned by a jet finding algorithm $[44,46]$ and the corresponding $p_{T}^{\text {miss }}$ are associated with each reconstructed vertex. The vertex with the largest value of $\Sigma p_{T}^{2}$ over the charged tracks and the $p_{T}^{\text {miss }}$ is taken to be the primary $p p$ interaction vertex. All isolation quantities are corrected for contributions from particles originating from pileup vertices. Electrons and muons must satisfy $\left|d_{z}\right|<0.1$ and $\left|d_{x y}\right|<0.05 \mathrm{~cm}$, where $d_{z}$ and $d_{x y}$ are the longitudinal and transverse impact parameters of leptons with respect to the primary vertex, respectively. For electrons in the ECAL end cap, we relax these requirements to $\left|d_{z}\right|<0.2$ and $\left|d_{x y}\right|<0.1 \mathrm{~cm}$.

For the two leading leptons we apply off-line $p_{T}$ thresholds of 25 and $15 \mathrm{GeV}$. Additional leptons must satisfy $p_{T}>10 \mathrm{GeV}$. With these thresholds, the trigger efficiency for trilepton events is close to $100 \%$. Events containing an opposite-sign same-flavor (OSSF) lepton pair with mass below $12 \mathrm{GeV}$ are vetoed to reduce background from low-mass resonances. We reject trilepton events containing an OSSF pair with mass below $81 \mathrm{GeV}$ when the trilepton mass is within a $Z$ boson mass window $(91 \pm 10 \mathrm{GeV})$. This suppresses events from the $Z \rightarrow \ell \ell^{*} \rightarrow \ell \ell \gamma$ background process, where the photon converts into two additional leptons, one of which is lost.

We classify the selected multilepton events into statistically independent search channels using the number of leptons and OSSF pairs, and other kinematic quantities as described below. The number of OSSF pairs is counted using each lepton once. For example, $\mu^{+} \mu^{+} e^{-}$is tagged as OSSF0, while $\mu^{+} \mu^{-} e^{+} e^{-}$is tagged as OSSF2. We further classify events as "on $Z$," "below $Z$," and "above $Z$," based 
on the presence and mass of at least one OSSF pair relative to a $Z$ boson mass window, defined as the range $81-101 \mathrm{GeV}$. In cases of ambiguity (such as $\mu^{+} \mu^{-} \mu^{-}$), the OSSF pair with its mass closest to that of the $Z$ boson takes precedence. We define $L_{T}$ as the scalar sum of all charged lepton $p_{T}$ 's, and we use $L_{T}+p_{T}^{\text {miss }}$ as the main discriminating variable. This choice allows us to maintain sensitivity to decay modes such as $\Sigma^{ \pm} \rightarrow \ell^{ \pm} Z \rightarrow \ell^{ \pm} \ell^{ \pm} \ell^{\mp}$ with large charged-lepton momenta, as well as $\Sigma^{0} \rightarrow \mathrm{H} \nu \rightarrow W W \nu$, where charged lepton $p_{T}$ 's are lower but the neutrinos contribute to $p_{T}^{\text {miss }}$. For events with OSSF1 and on $Z$, the sensitivity is improved by considering the transverse mass, $M_{T}=\left\{2 p_{T}^{\text {miss }} p_{T}^{\ell}[1-\right.$ $\left.\left.\cos \left(\vec{p}_{T}^{\text {miss }}, \vec{p}_{T}^{\ell}\right)\right]\right\}^{1 / 2}$ using the lepton that is not part of the OSSF pair. Events are grouped in eight bins of width 150 and $100 \mathrm{GeV}$ for $L_{T}+p_{T}^{\text {miss }}$ and $M_{T}$, respectively. In each case the highest bin includes overflow events.

Altogether, six event categories are considered with eight bins of $L_{T}+p_{T}^{\text {miss }}$ in (i) three leptons, OSSF1, above $Z$; (ii) three leptons, OSSF1, below $Z$; (iii) three leptons, OSSF0; (iv) four or more leptons, OSSF1; (v) four or more leptons, OSSF2; and eight bins of $M_{T}$ in (vi) three leptons, OSSF1, on $Z$. The 48 mutually exclusive signal regions are summarized in Table I.

The irreducible backgrounds are estimated using simulation samples. The dominant processes are $W Z$ and $Z Z$ production and are normalized to data using dedicated control regions. The $W Z$ control sample consists of events with exactly three leptons, tagged as OSSF1 on $Z$, satisfying $50<p_{T}^{\text {miss }}<100$ and $M_{T}>30 \mathrm{GeV}$. The ratio of the predicted $W Z$ rate to data (after correcting for backgrounds other than $W Z$ ) is $1.15 \pm 0.08$, where the uncertainty includes statistical and systematic contributions. The $Z Z$ control sample consists of events with exactly four leptons, OSSF2, on $Z$ for both pairs, and with $p_{T}^{\text {miss }}<50 \mathrm{GeV}$. The ratio of the predicted $Z Z$ rate to data is $1.25 \pm 0.06$. These factors are used to normalize the $W Z$ and $Z Z$ background estimates. We also use these control regions to verify the modeling of kinematic distributions such as $L_{T}+p_{T}^{\text {miss }}$ and $M_{T}$. The "rare" irreducible backgrounds from triboson, $t \bar{t} W$,

TABLE I. The signal regions summarized in terms of the number of leptons, the presence of an OSSF pair, and the kinematic variable used for signal discrimination. Each region is divided into eight bins in the kinematic variable, leading to 48 independent signal regions. Additional criteria based on $p_{T}^{\text {miss }}$ ensure that signal and control regions do not overlap.

\begin{tabular}{lccc}
\hline \hline$N_{\text {leptons }}$ & OSSF and mass & Variable & $p_{T}^{\text {miss }}$ requirement \\
\hline 3 & OSSF1, on $Z$ & $M_{T}$ & $p_{T}^{\text {miss }}>100 \mathrm{GeV}$ \\
& OSSF1, above $Z$ & $L_{T}+p_{T}^{\text {miss }}$ & no requirement \\
3 & OSSF1, below $Z$ & $L_{T}+p_{T}^{\text {miss }}$ & $p_{T}^{\text {miss }}>50 \mathrm{GeV}$ \\
& OSSF0 & $L_{T}+p_{T}^{\text {miss }}$ & no requirement \\
& OSSF1 & $L_{T}+p_{T}^{\text {miss }}$ & no requirement \\
$\geq 4$ & OSSF2 & $L_{T}+p_{T}^{\text {miss }}$ & $p_{T}^{\text {miss }}>50 \mathrm{GeV}$ if on $Z$ \\
\hline \hline
\end{tabular}

$t \bar{t} Z$, and Higgs boson processes are estimated from simulated samples using the theoretical cross sections.

The misidentified lepton background is estimated from observations using a three-dimensional implementation of a matrix method [47] in which probabilities (rates) of prompt and misidentified leptons satisfying a loose lepton selection to also pass a tight lepton selection are measured in data using a dilepton selection for prompt leptons, and a trilepton signal-depleted selection (OSSF1, on Z, $p_{T}^{\text {miss }}<50 \mathrm{GeV}$ ) for misidentified leptons. The rates are parametrized as a function of lepton $p_{T}, \eta$ and the number of PF candidates around the lepton. The rate measurements are dominated by $Z+$ jets events, and are corrected using simulation to an average of $Z+$ jets and $t \bar{t}+$ jets events.

A residual "conversion" background is also estimated using the data. When a final-state radiation photon from a lepton converts asymmetrically, often only one of the resultant leptons is reconstructed in the detector. To estimate this background, we count trilepton events where the trilepton mass is on $Z$, but the dilepton mass is below $Z$. These events are compared with dilepton + photon events where the dilepton invariant mass is below $Z$, but the combined invariant mass of the two leptons and the photon is on $Z$. Photons suitable for modeling conversions are selected by ignoring photons that are far away from a lepton or are close to a jet. The photon conversion rates are 3\% to 5\% depending upon the lepton type and $p_{T}$. The conversion background in a signal region is estimated by scaling the observed number of dilepton + photon events with these rates.

As an example of the complete background estimation, Fig. 1 shows the background predictions and the observed events with three leptons, OSSF1, above $Z$, and for events with four or more leptons, OSSF1, along with systematic uncertainties. The $W Z(Z Z)$ background has a systematic uncertainty of 7\% (5\%) arising from the normalization factor measurement. The rare backgrounds have an uncertainty of $50 \%$ in the theoretical cross section and $2.5 \%$ due to the integrated luminosity measurement. The misidentified background has an uncertainty of $30 \%$, which is derived by varying the measured rates up and down for each lepton flavor within their respective uncertainties. This uncertainty is dominated by the differences in rates in $Z+$ jets and $t \bar{t}+$ jets events. The conversion background has an uncertainty of $30 \%$ arising from contamination by nonconversion backgrounds in the control sample used to measure the conversion rate.

Corrections are applied to the simulated background events to account for differences from data in lepton identification and isolation efficiencies, dilepton trigger efficiencies, as well as in the overall scale and resolution of energy and momentum measurements for electrons, muons, and jets. The minor uncertainties due to these corrections are considered separately and result in a 1\%-10\% variation of the simulationbased background yields across all signal regions.

The uncertainties in the signal acceptance due to the PDF uncertainties are estimated by utilizing the replica weights 

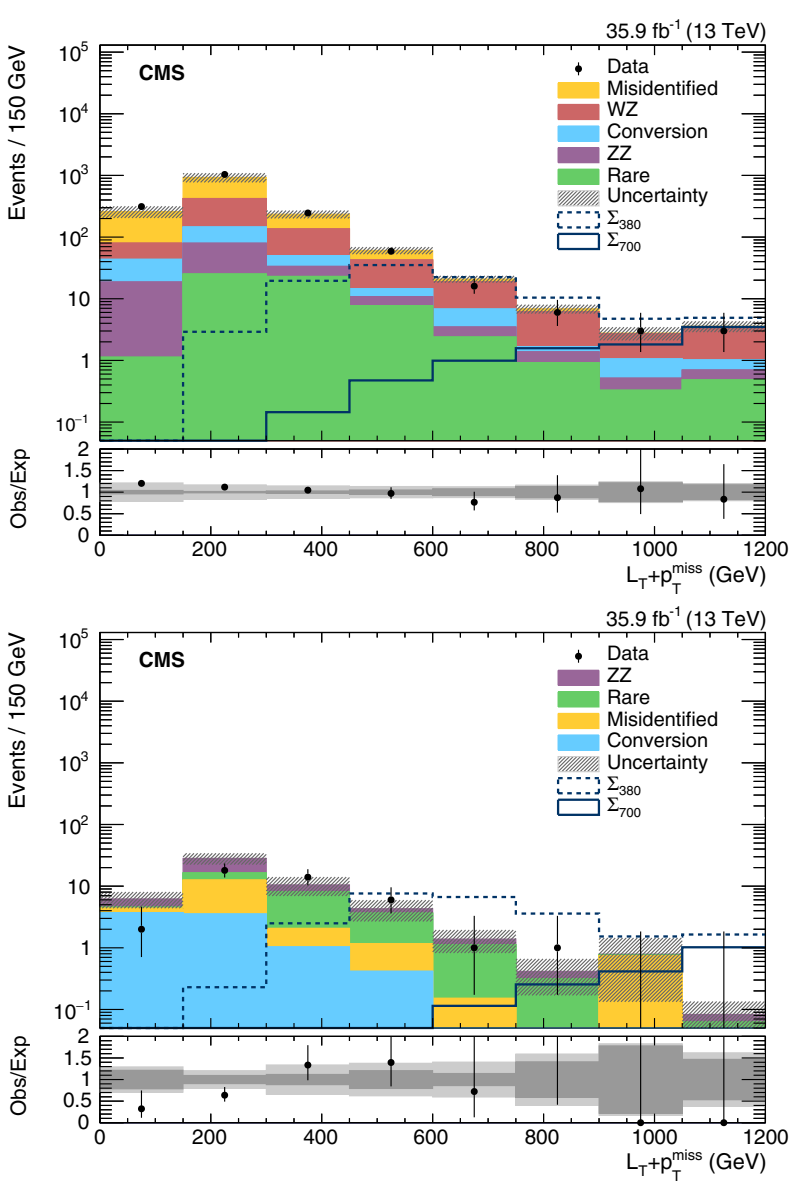

FIG. 1. The $L_{T}+p_{T}^{\text {miss }}$ distribution for events with three leptons and one OSSF pair with mass above $Z$ (upper) and the $L_{T}+p_{T}^{\text {miss }}$ distribution for events with four or more leptons and one OSSF pair (lower). The total SM background is shown as a stacked histogram of all contributing processes. The predictions for signal models (sum of all production and decay modes) with $m_{\Sigma}=700$ (solid line) and $m_{\Sigma}=380 \mathrm{GeV}$ (dashed line) are also shown. The lower panels show the ratio of observed to expected events. The hatched gray band in the upper panel, and the dark and light gray bands in the lower panel represent the total, statistical, and systematic uncertainties in the expected background, respectively.

of the NNPDF30_lo_as_0130_nf_4 PDF set [48], and are found to be less than $3 \%$ in all signal bins. The signal yields also have uncertainties due to the integrated luminosity measurement $(2.5 \%)$ and the corrections applied to the simulation samples mentioned in the previous paragraph.

We observe no statistically significant excess in the various signal regions that we probe. We calculate a 95\% confidence level (CL) upper limit on the sum of cross sections for the production of heavy fermion pairs $\left(\Sigma^{0} \Sigma^{+}, \Sigma^{0} \Sigma^{-}\right.$, and $\left.\Sigma^{+} \Sigma^{-}\right)$using the $\mathrm{CL}_{s}$ criterion [49-52]. We consider all sources of systematic and statistical uncertainties as nuisance parameters, with log-normal and gamma prior distributions, respectively, and perform a simultaneous fit across all search regions. Figure 2 shows the upper limits on the cross section in the flavordemocratic scenario where the observed (expected) limit

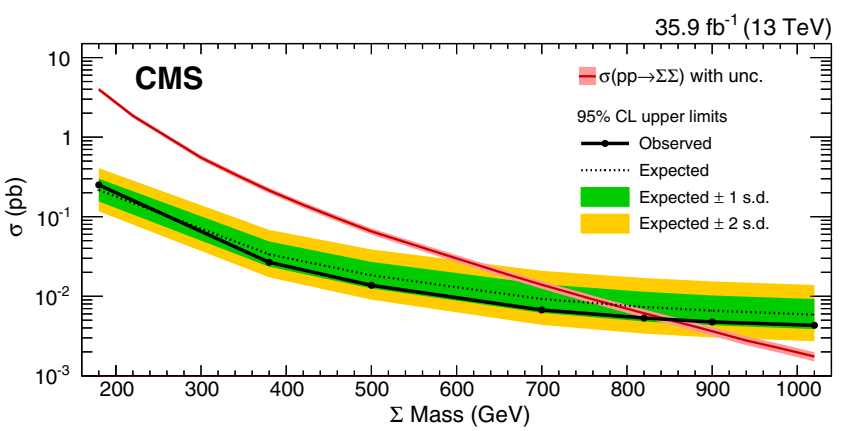

FIG. 2. The $95 \%$ confidence level upper limits on the cross section for production of heavy fermion pairs $\left(\Sigma^{0} \Sigma^{+}, \Sigma^{0} \Sigma^{-}\right.$, and $\Sigma^{+} \Sigma^{-}$). Also shown is the theoretical prediction for the cross section of the $\Sigma$ pair production via the type-III seesaw mechanism, with its uncertainty. In the flavor-democratic scenario $\left(B_{e}=B_{\mu}=B_{\tau}\right)$, heavy fermion pair production is excluded for masses below $840 \mathrm{GeV}$.

on masses of heavy fermions is $840(780) \mathrm{GeV}$. The signal cross section has an uncertainty of approximately 5\%-15\% due to choices of PDFs, and factorization and renormalization scales in the mass range we consider. Figure 3 shows the expected and observed excluded mass limits in the $B_{e}-B_{\tau}$ plane [note that $B_{\mu}=1-\left(B_{e}+B_{\tau}\right)$ ]. The limits are the most restrictive when the $\Sigma$ branching fraction to either electron or muon flavor is maximal, and become less restrictive as the branching fraction to $\tau$ flavor increases.
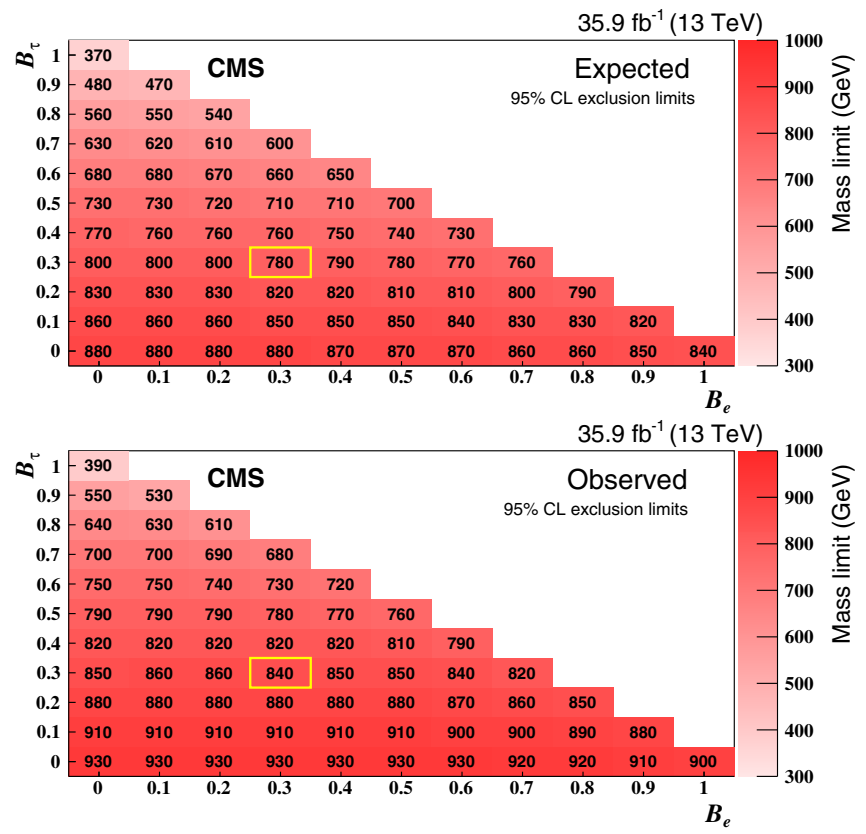

FIG. 3. The 95\% confidence level expected (upper) and observed (lower) limits on the heavy fermion mass $m_{\Sigma}(\mathrm{GeV})$ for each combination of branching fractions to the individual lepton flavors. The color scale represents the mass exclusion limit obtained at each point. The branching fraction to $\mu$ can be obtained from $B_{\mu}=1-\left[B_{e}+B_{\tau}\right]$. The highlighted box indicates the limit on $m_{\Sigma}$ for the flavor-democratic scenario $\left(B_{e}=B_{\mu}=B_{\tau}\right)$. 
In summary, a search has been performed for type-III seesaw heavy fermions in multilepton final states using $35.9 \mathrm{fb}^{-1}$ of $p p$ collision data at $\sqrt{s}=13 \mathrm{TeV}$, collected using the CMS detector. No significant discrepancies are observed between the data and the standard model prediction. Assuming degenerate heavy fermion masses, previously unexplored regions of the signal model as a function of the branching ratio of heavy fermions to each lepton flavor are excluded. In the lepton-flavor democratic scenario $\left(B_{e}=B_{\mu}=B_{\tau}\right)$, heavy fermions with mass below $840 \mathrm{GeV}$ are excluded at $95 \%$ confidence level. In the $\tau$-phobic case $\left(B_{\tau}=0, B_{e}+B_{\mu}=1\right)$ the mass limits range from $900 \mathrm{GeV}$ in the pure electron scenario to $930 \mathrm{GeV}$ in the pure muon scenario. In the electron- or muon-phobic case $\left(B_{\tau}=1, B_{e}+B_{\mu}=0\right)$ the mass limit is $390 \mathrm{GeV}$. These are the strongest constraints to date on the mass of heavy fermions associated with the type-III seesaw mechanism.

We congratulate our colleagues in the CERN accelerator departments for the excellent performance of the LHC and thank the technical and administrative staffs at CERN and at other CMS institutes for their contributions to the success of the CMS effort. In addition, we gratefully acknowledge the computing centers and personnel of the Worldwide LHC Computing Grid for delivering so effectively the computing infrastructure essential to our analyses. Finally, we acknowledge the enduring support for the construction and operation of the LHC and the CMS detector provided by the following funding agencies: BMWFW and FWF (Austria); FNRS and FWO (Belgium); CNPq, CAPES, FAPERJ, and FAPESP (Brazil); MES (Bulgaria); CERN; CAS, MoST, and NSFC (China); COLCIENCIAS (Colombia); MSES and CSF (Croatia); RPF (Cyprus); SENESCYT (Ecuador); MoER, ERC IUT, and ERDF (Estonia); Academy of Finland, MEC, and HIP (Finland); CEA and CNRS/IN2P3 (France); BMBF, DFG, and HGF (Germany); GSRT (Greece); OTKA and NIH (Hungary); DAE and DST (India); IPM (Iran); SFI (Ireland); INFN (Italy); MSIP and NRF (Republic of Korea); LAS (Lithuania); MOE and UM (Malaysia); BUAP, CINVESTAV, CONACYT, LNS, SEP, and UASLP-FAI (Mexico); MBIE (New Zealand); PAEC (Pakistan); MSHE and NSC (Poland); FCT (Portugal); JINR (Dubna); MON, RosAtom, RAS, RFBR and RAEP (Russia); MESTD (Serbia); SEIDI, CPAN, PCTI and FEDER (Spain); Swiss Funding Agencies (Switzerland); MST (Taipei); ThEPCenter, IPST, STAR, and NSTDA (Thailand); TUBITAK and TAEK (Turkey); NASU and SFFR (Ukraine); STFC (United Kingdom); DOE and NSF (USA).

[1] F. Capozzi, G. L. Fogli, E. Lisi, A. Marrone, D. Montanino, and A. Palazzo, Status of three-neutrino oscillation parameters, circa 2013, Phys. Rev. D 89, 093018 (2014).
[2] P. Minkowski, $\mu \rightarrow e \gamma$ at a rate of one out of $10^{9}$ muon decays?, Phys. Lett. 67B, 421 (1977).

[3] R. N. Mohapatra and Goran Senjanoviç, Neutrino Mass and Spontaneous Parity Nonconservation, Phys. Rev. Lett. 44, 912 (1980).

[4] M. Magg and C. Wetterich, Neutrino mass problem and gauge hierarchy, Phys. Lett. B 94, 61 (1980).

[5] R. N. Mohapatra and G. Senjanovic, Neutrino masses and mixings in gauge models with spontaneous parity violation, Phys. Rev. D 23, 165 (1981).

[6] J. Schechter and J. W. F. Valle, Neutrino masses in $\mathrm{SU}(2) \otimes$ U(1) theories, Phys. Rev. D 22, 2227 (1980).

[7] J. Schechter and J. W. F. Valle, Neutrino decay and spontaneous violation of lepton number, Phys. Rev. D 25, 774 (1982).

[8] R. Foot, H. Lew, X. G. He, and G. C. Joshi, See-saw neutrino masses induced by a triplet of leptons, Z. Phys. C 44, 441 (1989).

[9] R. N. Mohapatra, Mechanism for Understanding Small Neutrino Mass in Superstring Theories, Phys. Rev. Lett. 56, 561 (1986).

[10] R. N. Mohapatra and J. W. F. Valle, Neutrino mass and baryon-number nonconservation in superstring models, Phys. Rev. D 34, 1642 (1986).

[11] W. Wang and Z.-L. Han, Naturally small Dirac neutrino mass with intermediate $S U(2)_{L}$ multiplet fields, J. High Energy Phys. 04 (2017) 166.

[12] T. Li and X.-G. He, Neutrino masses and heavy triplet leptons at the LHC: Testability of type III seesaw, Phys. Rev. D 80, 093003 (2009).

[13] F. F. Deppisch, P. S. Bhupal Dev, and A. Pilaftsis, Neutrinos and collider physics, New J. Phys. 17, 075019 (2015).

[14] P. F. Perez, Type III seesaw and left-right symmetry, J. High Energy Phys. 03 (2009) 142.

[15] A. Arhrib, B. Bajc, D. K. Ghosh, T. Han, G.-Y. Huang, I. Puljak, and G. Senjanovic, Collider signatures for the heavy lepton triplet in the type I + III seesaw mechanism, Phys. Rev. D 82, 053004 (2010).

[16] F. del Aguila and J. A. Aguilar-Saavedra, Electroweak scale seesaw and heavy Dirac neutrino signals at LHC, Phys. Lett. B 672, 158 (2009).

[17] R. Franceschini, T. Hambye, and A. Strumia, Type-III seesaw mechanism at CERN LHC, Phys. Rev. D 78, 033002 (2008).

[18] K. L. McDonald, Probing exotic fermions from a seesaw/ radiative model at the LHC, J. High Energy Phys. 11 (2013) 131.

[19] R. Ding, Z.-L. Han, Y. Liao, H.-J. Liu, and J.-Y. Liu, Phenomenology in the minimal cascade seesaw mechanism for neutrino masses, Phys. Rev. D 89, 115024 (2014).

[20] F. del Aguila and J. A. Aguilar-Saavedra, Distinguishing seesaw models at LHC with multi-lepton signals, Nucl. Phys. B813, 22 (2009).

[21] F. del Aguila, J. A. Aguilar-Saavedra, and J. de Blas, Trilepton signals: the golden channel for seesaw searches at LHC, Acta Phys. Pol. B 40, 2901 (2009).

[22] C. Biggio and F. Bonnet, Implementation of the Type III seesaw model in FeynRules/MadGraph and prospects for discovery with early LHC data, Eur. Phys. J. C 72, 1899 (2012). 
[23] CMS Collaboration, Search for heavy lepton partners of neutrinos in proton-proton collisions in the context of the type III seesaw mechanism, Phys. Lett. B 718, 348 (2012).

[24] ATLAS Collaboration, Search for type-III seesaw heavy leptons in $p p$ collisions at $\sqrt{s}=8 \mathrm{TeV}$ with the ATLAS detector, Phys. Lett. D 92, 032001 (2015).

[25] CMS Collaboration, The CMS experiment at the CERN LHC, J. Instrum. 3, S08004 (2008).

[26] CMS Collaboration, The CMS trigger system, J. Instrum. 12, P01020 (2017).

[27] J. Alwall, R. Frederix, S. Frixione, V. Hirschi, F. Maltoni, O. Mattelaer, H.-S. Shao, T. Stelzer, P. Torrielli, and M. Zaro, The automated computation of tree-level and next-to-leading order differential cross sections, and their matching to parton shower simulations, J. High Energy Phys. 07 (2014) 079.

[28] R. D. Ball et al. (NNPDF), Parton distributions for the LHC Run II, J. High Energy Phys. 04 (2015) 040.

[29] B. Fuks, M. Klasen, D. R. Lamprea, and M. Rothering, Gaugino production in proton-proton collisions at a centerof-mass energy of $8 \mathrm{TeV}$, J. High Energy Phys. 10 (2012) 081.

[30] B. Fuks, M. Klasen, D. R. Lamprea, and M. Rothering, Precision predictions for electroweak superpartner production at hadron colliders with Resummino, Eur. Phys. J. C 73, 2480 (2013).

[31] P. Nason, A new method for combining NLO QCD with shower Monte Carlo algorithms, J. High Energy Phys. 11 (2004) 040.

[32] S. Frixione, P. Nason, and C. Oleari, Matching NLO QCD computations with parton shower simulations: the POWHEG method, J. High Energy Phys. 11 (2007) 070.

[33] S. Alioli, P. Nason, C. Oleari, and E. Re, A general framework for implementing NLO calculations in shower Monte Carlo programs: the POWHEG BOX, J. High Energy Phys. 06 (2010) 043.

[34] Y. Gao, A. V. Gritsan, Z. Guo, K. Melnikov, M. Schulze, and N. V. Tran, Spin determination of single-produced resonances at hadron colliders, Phys. Rev. D 81, 075022 (2010).

[35] S. Bolognesi, Y. Gao, A. V. Gritsan, K. Melnikov, M. Schulze, N. V. Tran, and A. Whitbeck, On the spin and parity of a single-produced resonance at the LHC, Phys. Rev. D 86, 095031 (2012).

[36] I. Anderson, S. Bolognesi, F. Caola, Y. Gao, A. V. Gritsan, C. B. Martin, K. Melnikov, M. Schulze, N. V. Tran, A. Whitbeck, and Y. Zhou, Constraining anomalous $H V V$ interactions at proton and lepton colliders, Phys. Rev. D 89, 035007 (2014).

[37] A. V. Gritsan, R. Röntsch, M. Schulze, and M. Xiao, Constraining anomalous Higgs boson couplings to the heavy flavor fermions using matrix element techniques, Phys. Rev. D 94, 055023 (2016).

[38] T. Sjöstrand, S. Mrenna, and P.Z. Skands, PYTHIA 6.4 physics and manual, J. High Energy Phys. 05 (2006) 026.

[39] T. Sjöstrand, S. Ask, J. R. Christiansen, R. Corke, N. Desai, P. Ilten, S. Mrenna, S. Prestel, C. O. Rasmussen, and P. Z. Skands, An introduction to PYTHIA 8.2, Comput. Phys. Commun. 191, 159 (2015).

[40] S. Agostinelli et al. (GEANT4), GEANT4-a simulation toolkit, Nucl. Instrum. Methods Phys. Res., Sect. A 506, 250 (2003).

[41] CMS Collaboration, CMS Physics Analysis Summary CMS-PAS-JME-14-001, 2014.

[42] CMS Collaboration, Performance of electron reconstruction and selection with the CMS detector in proton-proton collisions at $\sqrt{s}=8 \mathrm{TeV}$, J. Instrum. 10, P06005 (2015).

[43] CMS Collaboration, Particle-flow reconstruction and global event description with the CMS detector, J. Instrum. 12, P10003 (2017).

[44] M. Cacciari, G. P. Salam, and G. Soyez, The anti- $k_{t}$ jet clustering algorithm, J. High Energy Phys. 04 (2008) 063.

[45] CMS Collaboration, Performance of the CMS missing transverse momentum reconstruction in $p p$ data at $\sqrt{s}=8 \mathrm{TeV}$, J. Instrum. 10, P02006 (2015).

[46] M. Cacciari, G. P. Salam, and G. Soyez, FastJet user manual, Eur. Phys. J. C 72, 1896 (2012).

[47] CMS Collaboration, Measurement of the $t \bar{t}$ production cross section in the dilepton channel in $p p$ collisions at $\sqrt{s}=7 \mathrm{TeV}$, J. High Energy Phys. 11 (2012) 067.

[48] J. Butterworth et al., PDF4LHC recommendations for LHC Run II, J. Phys. G 43, 023001 (2016).

[49] T. Junk, Confidence level computation for combining searches with small statistics, Nucl. Instrum. Methods Phys. Res., Sect. A 434, 435 (1999).

[50] A. L. Read, Presentation of search results: The $C L_{s}$ technique, J. Phys. G 28, 2693 (2002).

[51] ATLAS and CMS Collaborations, Technical Report No. CMS NOTE-2011/005 and No. ATL-PHYS-PUB2011-11, 2011.

[52] G. Cowan, K. Cranmer, E. Gross, and O. Vitells, Asymptotic formulae for likelihood-based tests of new physics, Eur. Phys. J. C 71, 1554 (2011); Erratum, Eur. Phys. J. C 73, 2501(E) (2013).

A. M. Sirunyan, ${ }^{1}$ A. Tumasyan, ${ }^{1}$ W. Adam, ${ }^{2}$ F. Ambrogi, ${ }^{2}$ E. Asilar, ${ }^{2}$ T. Bergauer, ${ }^{2}$ J. Brandstetter, ${ }^{2}$ E. Brondolin, ${ }^{2}$ M. Dragicevic, ${ }^{2}$ J. Erö, ${ }^{2}$ M. Flechl, ${ }^{2}$ M. Friedl, ${ }^{2}$ R. Frühwirth, ${ }^{2, b}$ V. M. Ghete, ${ }^{2}$ J. Grossmann, ${ }^{2}$ J. Hrubec, ${ }^{2}$ M. Jeitler, ${ }^{2, b}$ A. König, ${ }^{2}$ N. Krammer, ${ }^{2}$ I. Krätschmer, ${ }^{2}$ D. Liko, ${ }^{2}$ T. Madlener, ${ }^{2}$ I. Mikulec, ${ }^{2}$ E. Pree, ${ }^{2}$ D. Rabady, ${ }^{2}$ N. Rad,${ }^{2}$ H. Rohringer, ${ }^{2}$ J. Schieck, ${ }^{2, b}$ R. Schöfbeck, ${ }^{2}$ M. Spanring, ${ }^{2}$ D. Spitzbart, ${ }^{2}$ W. Waltenberger, ${ }^{2}$ J. Wittmann, ${ }^{2}$ C.-E. Wulz, ${ }^{2, b}$ M. Zarucki, ${ }^{2}$ V. Chekhovsky, ${ }^{3}$ V. Mossolov, ${ }^{3}$ J. Suarez Gonzalez, ${ }^{3}$ E. A. De Wolf, ${ }^{4}$ D. Di Croce, ${ }^{4}$ X. Janssen, ${ }^{4}$ J. Lauwers, ${ }^{4}$ H. Van Haevermaet, ${ }^{4}$ P. Van Mechelen, ${ }^{4}$ N. Van Remortel, ${ }^{4}$ S. Abu Zeid, ${ }_{5}^{5}$ F. Blekman, ${ }^{5}$ J. D'Hondt, ${ }^{5}$ I. De Bruyn, ${ }^{5}$ J. De Clercq, ${ }^{5}$ K. Deroover, ${ }^{5}$ G. Flouris, ${ }^{5}$ D. Lontkovskyi, ${ }^{5}$ S. Lowette, ${ }^{5}$ S. Moortgat, ${ }^{5}$ L. Moreels, ${ }^{5}$ Q. Python, 
K. Skovpen, ${ }^{5}$ S. Tavernier, ${ }^{5}$ W. Van Doninck, ${ }^{5}$ P. Van Mulders, ${ }^{5}$ I. Van Parijs, ${ }^{5}$ H. Brun, ${ }^{6}$ B. Clerbaux, ${ }^{6}$ G. De Lentdecker, ${ }^{6}$ H. Delannoy, ${ }^{6}$ G. Fasanella, ${ }^{6}$ L. Favart, ${ }^{6}$ R. Goldouzian, ${ }^{6}$ A. Grebenyuk, ${ }^{6}$ G. Karapostoli, ${ }^{6}$ T. Lenzi, ${ }^{6}$ J. Luetic, ${ }^{6}$ T. Maerschalk, ${ }^{6}$ A. Marinov, ${ }^{6}$ A. Randle-conde, ${ }^{6}$ T. Seva, ${ }^{6}$ C. Vander Velde, ${ }^{6}$ P. Vanlaer, ${ }^{6}$ D. Vannerom, ${ }^{6}$ R. Yonamine, ${ }^{6}$ F. Zenoni, ${ }^{6}$ F. Zhang, ${ }^{6, c}$ A. Cimmino, ${ }^{7}$ T. Cornelis, ${ }^{7}$ D. Dobur, ${ }^{7}$ A. Fagot, ${ }^{7}$ M. Gul, ${ }^{7}$ I. Khvastunov, ${ }^{7}$ D. Poyraz, ${ }^{7}$ C. Roskas, ${ }^{7}$ S. Salva, ${ }^{7}$ M. Tytgat, ${ }^{7}$ W. Verbeke, ${ }^{7}$ N. Zaganidis, ${ }^{7}$ H. Bakhshiansohi, ${ }^{8}$ O. Bondu, ${ }^{8}$ S. Brochet, ${ }^{8}$ G. Bruno, ${ }^{8}$ A. Caudron, ${ }^{8}$

S. De Visscher, ${ }^{8}$ C. Delaere, ${ }^{8}$ M. Delcourt, ${ }^{8}$ B. Francois, ${ }^{8}$ A. Giammanco, ${ }^{8}$ A. Jafari, ${ }^{8}$ M. Komm, ${ }^{8}$ G. Krintiras, ${ }^{8}$ V. Lemaitre, ${ }^{8}$ A. Magitteri, ${ }^{8}$ A. Mertens, ${ }^{8}$ M. Musich, ${ }^{8}$ K. Piotrzkowski, ${ }^{8}$ L. Quertenmont, ${ }^{8}$ M. Vidal Marono, ${ }^{8}$ S. Wertz, ${ }^{8}$ N. Beliy, ${ }^{9}$ W. L. Aldá Júnior, ${ }^{10}$ F. L. Alves,${ }^{10}$ G. A. Alves,${ }^{10}$ L. Brito, ${ }^{10}$ M. Correa Martins Junior, ${ }^{10}$ C. Hensel, ${ }^{10}$ A. Moraes, ${ }^{10}$ M. E. Pol, ${ }^{10}$ P. Rebello Teles,${ }^{10}$ E. Belchior Batista Das Chagas,${ }^{11}$ W. Carvalho, ${ }^{11}$ J. Chinellato, ${ }^{11, d}$ A. Custódio, ${ }^{11}$ E. M. Da Costa, ${ }^{11}$ G. G. Da Silveira, ${ }^{11, e}$ D. De Jesus Damiao, ${ }^{11}$ S. Fonseca De Souza, ${ }^{11}$

L. M. Huertas Guativa, ${ }^{11}$ H. Malbouisson, ${ }^{11}$ M. Melo De Almeida, ${ }^{11}$ C. Mora Herrera, ${ }^{11}$ L. Mundim, ${ }^{11}$ H. Nogima, ${ }^{11}$ A. Santoro, ${ }^{11}$ A. Sznajder, ${ }^{11}$ E. J. Tonelli Manganote, ${ }^{11, d}$ F. Torres Da Silva De Araujo, ${ }^{11}$ A. Vilela Pereira, ${ }^{11}$ S. Ahuja, ${ }^{12 a}$ C. A. Bernardes, ${ }^{12 a}$ T. R. Fernandez Perez Tomei, ${ }^{12 a}$ E. M. Gregores, ${ }^{12 b}$ P. G. Mercadante, ${ }^{12 b}$ S. F. Novaes, ${ }^{12 a}$ Sandra S. Padula, ${ }^{12 \mathrm{a}}$ D. Romero Abad, ${ }^{12 \mathrm{~b}}$ J. C. Ruiz Vargas, ${ }^{12 \mathrm{a}}$ A. Aleksandrov, ${ }^{13}$ R. Hadjiiska, ${ }^{13}$ P. Iaydjiev, ${ }^{13}$ M. Misheva, ${ }^{13}$ M. Rodozov, ${ }^{13}$ M. Shopova, ${ }^{13}$ S. Stoykova, ${ }^{13}$ G. Sultanov, ${ }^{13}$ A. Dimitrov,${ }^{14}$ I. Glushkov, ${ }^{14}$ L. Litov, ${ }^{14}$ B. Pavlov ${ }^{14}$ P. Petkov, ${ }^{14}$ W. Fang,${ }^{15, f}$ X. Gao, ${ }^{15, f}$ M. Ahmad, ${ }^{16}$ J. G. Bian, ${ }^{16}$ G. M. Chen, ${ }^{16}$ H. S. Chen ${ }^{16}$ M. Chen, ${ }^{16}$ Y. Chen, ${ }^{16}$ C. H. Jiang, ${ }^{16}$ D. Leggat,${ }^{16}$ H. Liao, ${ }^{16}$ Z. Liu, ${ }^{16}$ F. Romeo, ${ }^{16}$ S. M. Shaheen, ${ }^{16}$ A. Spiezia, ${ }^{16}$ J. Tao, ${ }^{16}$ C. Wang,${ }^{16}$ Z. Wang, ${ }^{16}$ E. Yazgan, ${ }^{16}$ H. Zhang, ${ }^{16}$ J. Zhao, ${ }^{16}$ Y. Ban,${ }^{17}$ G. Chen, ${ }^{17}$ Q. Li, ${ }^{17}$ S. Liu, ${ }^{17}$ Y. Mao, ${ }^{17}$ S. J. Qian, ${ }^{17}$ D. Wang,${ }^{17}$ Z. Xu, ${ }^{17}$ C. Avila, ${ }^{18}$ A. Cabrera, ${ }^{18}$ L. F. Chaparro Sierra, ${ }^{18}$ C. Florez,${ }^{18}$ C. F. González Hernández, ${ }^{18}$ J. D. Ruiz Alvarez, ${ }^{18}$ B. Courbon, ${ }^{19}$ N. Godinovic, ${ }^{19}$ D. Lelas, ${ }^{19}$ I. Puljak, ${ }^{19}$ P. M. Ribeiro Cipriano, ${ }^{19}$ T. Sculac, ${ }^{19}$ Z. Antunovic,${ }^{20}$ M. Kovac, ${ }^{20}$ V. Brigljevic, ${ }^{21}$ D. Ferencek, ${ }^{21}$ K. Kadija, ${ }^{21}$ B. Mesic, ${ }^{21}$ A. Starodumov, ${ }^{21, g}$ T. Susa, ${ }^{21}$ M. W. Ather, ${ }^{22}$ A. Attikis, ${ }^{22}$ G. Mavromanolakis, ${ }^{22}$ J. Mousa, ${ }^{22}$ C. Nicolaou, ${ }^{22}$ F. Ptochos, ${ }^{22}$ P. A. Razis,${ }^{22}$ H. Rykaczewski, ${ }^{22}$ M. Finger, ${ }^{23, h}$ M. Finger Jr., ${ }^{23, h}$ E. Carrera Jarrin, ${ }^{24}$ E. El-khateeb, ${ }^{25, i}$ S. Elgammal, ${ }^{25, j}$ A. Ellithi Kamel, ${ }^{25, k}$ R. K. Dewanjee, ${ }^{26}$ M. Kadastik,${ }^{26}$ L. Perrini, ${ }^{26}$ M. Raidal, ${ }^{26}$ A. Tiko, ${ }^{26}$ C. Veelken, ${ }^{26}$ P. Eerola, ${ }^{27}$ J. Pekkanen, ${ }^{27}$ M. Voutilainen, ${ }^{27}$ J. Härkönen, ${ }^{28}$ T. Järvinen, ${ }^{28}$ V. Karimäki, ${ }^{28}$ R. Kinnunen, ${ }^{28}$ T. Lampén, ${ }^{28}$ K. Lassila-Perini, ${ }^{28}$ S. Lehti, ${ }^{28}$ T. Lindén, ${ }^{28}$ P. Luukka, ${ }^{28}$ E. Tuominen, ${ }^{28}$ J. Tuominiemi, ${ }^{28}$ E. Tuovinen, ${ }^{28}$ J. Talvitie, ${ }^{29}$ T. Tuuva, ${ }^{29}$ M. Besancon, ${ }^{30}$ F. Couderc ${ }^{30}$ M. Dejardin,${ }^{30}$ D. Denegri, ${ }^{30}$ J. L. Faure,${ }^{30}$ F. Ferri, ${ }^{30}$ S. Ganjour, ${ }^{30}$ S. Ghosh,${ }^{30}$ A. Givernaud,${ }^{30}$ P. Gras,${ }^{30}$ G. Hamel de Monchenault, ${ }^{30}$ P. Jarry,${ }^{30}$ I. Kucher, ${ }^{30}$ E. Locci, ${ }^{30}$ M. Machet,${ }^{30}$ J. Malcles, ${ }^{30}$ G. Negro, ${ }^{30}$ J. Rander, ${ }^{30}$ A. Rosowsky, ${ }^{30}$ M. Ö. Sahin, ${ }^{30}$ M. Titov ${ }^{30}$ A. Abdulsalam, ${ }^{31}$ I. Antropov,${ }^{31}$ S. Baffioni, ${ }^{31}$ F. Beaudette, ${ }^{31}$ P. Busson, ${ }^{31}$ L. Cadamuro, ${ }^{31}$ C. Charlot, ${ }^{31}$ R. Granier de Cassagnac, ${ }^{31}$ M. Jo, ${ }^{31}$ S. Lisniak, ${ }^{31}$ A. Lobanov, ${ }^{31}$ J. Martin Blanco, ${ }^{31}$ M. Nguyen, ${ }^{31}$ C. Ochando, ${ }^{31}$ G. Ortona, ${ }^{31}$ P. Paganini, ${ }^{31}$ P. Pigard, ${ }^{31}$ S. Regnard,${ }^{31}$ R. Salerno, ${ }^{31}$ J. B. Sauvan,${ }^{31}$ Y. Sirois,${ }^{31}$

A. G. Stahl Leiton, ${ }^{31}$ T. Strebler, ${ }^{31}$ Y. Yilmaz, ${ }^{31}$ A. Zabi, ${ }^{31}$ A. Zghiche, ${ }^{31}$ J.-L. Agram, ${ }^{32,1}$ J. Andrea, ${ }^{32}$ D. Bloch, ${ }^{32}$ J.-M. Brom, ${ }^{32}$ M. Buttignol, ${ }^{32}$ E. C. Chabert, ${ }^{32}$ N. Chanon, ${ }^{32}$ C. Collard, ${ }^{32}$ E. Conte, ${ }^{32,1}$ X. Coubez, ${ }^{32}$ J.-C. Fontaine, ${ }^{32,1}$ D. Gelé, ${ }^{32}$ U. Goerlach, ${ }^{32}$ M. Jansová, ${ }^{32}$ A.-C. Le Bihan, ${ }^{32}$ N. Tonon, ${ }^{32}$ P. Van Hove ${ }^{32}$ S. Gadrat ${ }^{33}$ S. Beauceron, ${ }^{34}$ C. Bernet, ${ }^{34}$ G. Boudoul, ${ }^{34}$ R. Chierici,${ }^{34}$ D. Contardo,${ }^{34}$ P. Depasse, ${ }^{34}$ H. El Mamouni,${ }^{34}$ J. Fay, ${ }^{34}$ L. Finco, ${ }^{34}$ S. Gascon,,${ }^{34}$ M. Gouzevitch, ${ }^{34}$ G. Grenier, ${ }^{34}$ B. Ille, ${ }^{34}$ F. Lagarde, ${ }^{34}$ I. B. Laktineh,${ }^{34}$ M. Lethuillier, ${ }^{34}$ L. Mirabito, ${ }^{34}$ A. L. Pequegnot, ${ }^{34}$ S. Perries ${ }^{34}$ A. Popov,${ }^{34, m}$ V. Sordini, ${ }^{34}$ M. Vander Donckt, ${ }^{34}$ S. Viret,${ }^{34}$ T. Toriashvili, ${ }^{35, n}$ D. Lomidze, ${ }^{36}$ C. Autermann, ${ }^{37}$ S. Beranek, ${ }^{37}$ L. Feld,${ }^{37}$ M. K. Kiesel, ${ }^{37}$ K. Klein,${ }^{37}$ M. Lipinski, ${ }^{37}$ M. Preuten, ${ }^{37}$ C. Schomakers,${ }^{37}$ J. Schulz,${ }^{37}$ T. Verlage, ${ }^{37}$ A. Albert, ${ }^{38}$ E. Dietz-Laursonn, ${ }^{38}$ D. Duchardt, ${ }^{38}$ M. Endres ${ }^{38}$ M. Erdmann, ${ }^{38}$ S. Erdweg, ${ }^{38}$ T. Esch,${ }^{38}$ R. Fischer, ${ }^{38}$ A. Güth, ${ }^{38}$ M. Hamer, ${ }^{38}$ T. Hebbeker, ${ }^{38}$ C. Heidemann, ${ }^{38}$ K. Hoepfner, ${ }^{38}$ S. Knutzen, ${ }^{38}$ M. Merschmeyer, ${ }^{38}$ A. Meyer, ${ }^{38}$ P. Millet, ${ }^{38}$ S. Mukherjee, ${ }^{38}$ M. Olschewski, ${ }^{38}$ K. Padeken, ${ }^{38}$ T. Pook, ${ }^{38}$ M. Radziej, ${ }^{38}$ H. Reithler, ${ }^{38}$ M. Rieger, ${ }^{38}$ F. Scheuch, ${ }^{38}$ D. Teyssier, ${ }^{38}$ S. Thüer, ${ }^{38}$ G. Flügge, ${ }^{39}$ B. Kargoll,,${ }^{39}$ T. Kress, ${ }^{39}$ A. Künsken, ${ }^{39}$ J. Lingemann, ${ }^{39}$ T. Müller,${ }^{39}$ A. Nehrkorn, ${ }^{39}$ A. Nowack, ${ }^{39}$ C. Pistone, ${ }^{39}$ O. Pooth, ${ }^{39}$ A. Stahl, ${ }^{39,0}$ M. Aldaya Martin, ${ }^{40}$ T. Arndt, ${ }^{40}$ C. Asawatangtrakuldee ${ }^{40}$ K. Beernaert, ${ }^{40}$ O. Behnke, ${ }^{40}$ U. Behrens, ${ }^{40}$ A. Bermúdez Martínez, ${ }^{40}$ A. A. Bin Anuar, ${ }^{40}$ K. Borras, ${ }^{40, p}$ V. Botta, ${ }^{40}$ A. Campbell,${ }^{40}$ P. Connor,${ }^{40}$ C. Contreras-Campana,${ }^{40}$ F. Costanza ${ }^{40}$ C. Diez Pardos, ${ }^{40}$ G. Eckerlin, ${ }^{40}$ D. Eckstein, ${ }^{40}$ T. Eichhorn, ${ }^{40}$ E. Eren, ${ }^{40}$ E. Gallo, ${ }^{40, q}$ J. Garay Garcia, ${ }^{40}$ A. Geiser ${ }^{40}$ A. Gizhko, ${ }^{40}$ J. M. Grados Luyando, ${ }^{40}$ A. Grohsjean, ${ }^{40}$ P. Gunnellini, ${ }^{40}$ A. Harb, ${ }^{40}$ J. Hauk, ${ }^{40}$ M. Hempel, ${ }^{40, r}$ H. Jung, ${ }^{40}$ A. Kalogeropoulos, ${ }^{40}$ M. Kasemann, ${ }^{40}$ J. Keaveney, ${ }^{40}$ C. Kleinwort, ${ }^{40}$ I. Korol, ${ }^{40}$ D. Krücker, ${ }^{40}$ W. Lange, ${ }^{40}$ A. Lelek, ${ }^{40}$ T. Lenz,${ }^{40}$ J. Leonard, ${ }^{40}$ K. Lipka, ${ }^{40}$ W. Lohmann, ${ }^{40, r}$ R. Mankel, ${ }^{40}$ I.-A. Melzer-Pellmann, ${ }^{40}$ A. B. Meyer, ${ }^{40}$ G. Mittag, ${ }^{40}$ 
J. Mnich, ${ }^{40}$ A. Mussgiller, ${ }^{40}$ E. Ntomari, ${ }^{40}$ D. Pitzl ${ }^{40}$ A. Raspereza, ${ }^{40}$ B. Roland ${ }^{40}$ M. Savitskyi, ${ }^{40}$ P. Saxena,${ }^{40}$ R. Shevchenko, ${ }^{40}$ S. Spannagel, ${ }^{40}$ N. Stefaniuk, ${ }^{40}$ G. P. Van Onsem, ${ }^{40}$ R. Walsh,${ }^{40}$ Y. Wen, ${ }^{40}$ K. Wichmann, ${ }^{40}$ C. Wissing, ${ }^{40}$ O. Zenaiev, ${ }^{40}$ S. Bein, ${ }^{41}$ V. Blobel, ${ }^{41}$ M. Centis Vignali, ${ }^{41}$ T. Dreyer, ${ }^{41}$ E. Garutti, ${ }^{41}$ D. Gonzalez, ${ }^{41}$ J. Haller, ${ }^{41}$ A. Hinzmann, ${ }^{41}$ M. Hoffmann, ${ }^{41}$ A. Karavdina, ${ }^{41}$ R. Klanner, ${ }^{41}$ R. Kogler, ${ }^{41}$ N. Kovalchuk, ${ }^{41}$ S. Kurz, ${ }^{41}$ T. Lapsien, ${ }^{41}$ I. Marchesini ${ }^{41}$ D. Marconi ${ }^{41}$ M. Meyer, ${ }^{41}$ M. Niedziela, ${ }^{41}$ D. Nowatschin,${ }^{41}$ F. Pantaleo, ${ }^{41,0}$ T. Peiffer, ${ }^{41}$ A. Perieanu, ${ }^{41}$ C. Scharf, ${ }^{41}$ P. Schleper, ${ }^{41}$ A. Schmidt, ${ }^{41}$ S. Schumann, ${ }^{41}$ J. Schwandt, ${ }^{41}$ J. Sonneveld, ${ }^{41}$ H. Stadie, ${ }^{41}$ G. Steinbrück, ${ }^{41}$ F. M. Stober ${ }^{41}$ M. Stöver ${ }^{41}$ H. Tholen, ${ }^{41}$ D. Troendle, ${ }^{41}$ E. Usai ${ }^{41}$ L. Vanelderen, ${ }^{41}$ A. Vanhoefer, ${ }^{41}$ B. Vormwald ${ }^{41}$ M. Akbiyik, ${ }^{42}$ C. Barth, ${ }^{42}$ S. Baur, ${ }^{42}$ E. Butz, ${ }^{42}$ R. Caspart, ${ }^{42}$ T. Chwalek,${ }^{42}$ F. Colombo, ${ }^{42}$ W. De Boer, ${ }^{42}$ A. Dierlamm, ${ }^{42}$ B. Freund,${ }^{42}$ R. Friese,${ }^{42}$ M. Giffels, ${ }^{42}$ A. Gilbert, ${ }^{42}$ D. Haitz ${ }^{42}$ F. Hartmann, ${ }^{42, o}$ S. M. Heindl, ${ }^{42}$ U. Husemann, ${ }^{42}$ F. Kassel, ${ }^{42, o}$ S. Kudella, ${ }^{42}$ H. Mildner, ${ }^{42}$ M. U. Mozer, ${ }^{42}$ Th. Müller, ${ }^{42}$ M. Plagge, ${ }^{42}$ G. Quast, ${ }^{42}$ K. Rabbertz ${ }^{42}$ M. Schröder, ${ }^{42}$ I. Shvetsov, ${ }^{42}$ G. Sieber, ${ }^{42}$ H. J. Simonis, ${ }^{42}$ R. Ulrich,${ }^{42}$ S. Wayand, ${ }^{42}$ M. Weber, ${ }^{42}$ T. Weiler, ${ }^{42}$ S. Williamson, ${ }^{42}$ C. Wöhrmann, ${ }^{42}$ R. Wolf, ${ }^{42}$ G. Anagnostou, ${ }^{43}$ G. Daskalakis, ${ }^{43}$ T. Geralis, ${ }^{43}$ V. A. Giakoumopoulou, ${ }^{43}$ A. Kyriakis, ${ }^{43}$ D. Loukas, ${ }^{43}$ I. Topsis-Giotis, ${ }^{43}$ G. Karathanasis, ${ }^{44}$ S. Kesisoglou, ${ }^{44}$ A. Panagiotou, ${ }^{44}$ N. Saoulidou, ${ }^{44}$ I. Evangelou, ${ }^{45}$ C. Foudas, ${ }^{45}$ P. Kokkas, ${ }^{45}$ S. Mallios, ${ }^{45}$ N. Manthos, ${ }^{45}$ I. Papadopoulos,${ }^{45}$ E. Paradas, ${ }^{45}$ J. Strologas, ${ }^{45}$ F. A. Triantis, ${ }^{45}$ M. Csanad ${ }^{46}$ N. Filipovic, ${ }^{46}$ G. Pasztor,${ }^{46}$ G. Bencze,${ }^{47}$ C. Hajdu,${ }^{47}$ D. Horvath, ${ }^{47, s}$ Á. Hunyadi,${ }^{47}$ F. Sikler,${ }^{47}$ V. Veszpremi ${ }^{47}$ G. Vesztergombi, ${ }^{47, t}$ A. J. Zsigmond, ${ }^{47}$ N. Beni, ${ }^{48}$ S. Czellar, ${ }^{48}$ J. Karancsi, ${ }^{48, u}$ A. Makovec, ${ }^{48}$ J. Molnar, ${ }^{48}$ Z. Szillasi, ${ }^{48}$ M. Bartók,${ }^{49, \mathrm{t}}$ P. Raics, ${ }^{49}$ Z. L. Trocsanyi, ${ }^{49}$ B. Ujvari, ${ }^{49}$ S. Choudhury, ${ }^{50}$ J. R. Komaragiri, ${ }^{50}$ S. Bahinipati, ${ }^{51, v}$ S. Bhowmik, ${ }^{51}$ P. Mal, ${ }^{51}$ K. Mandal,${ }^{51}$ A. Nayak, ${ }^{51, w}$ D. K. Sahoo, ${ }^{51, v}$ N. Sahoo,${ }^{51}$ S. K. Swain, ${ }^{51}$ S. Bansal, ${ }^{52}$ S. B. Beri, ${ }^{52}$ V. Bhatnagar, ${ }^{52}$ R. Chawla, ${ }^{52}$ N. Dhingra, ${ }^{52}$ A. K. Kalsi, ${ }^{52}$ A. Kaur, ${ }^{52}$ M. Kaur, ${ }^{52}$ R. Kumar, ${ }^{52}$ P. Kumari, ${ }^{52}$ A. Mehta, ${ }^{52}$ J. B. Singh, ${ }^{52}$ G. Walia, ${ }^{52}$ Ashok Kumar, ${ }^{53}$ Aashaq Shah, ${ }^{53}$ A. Bhardwaj, ${ }^{53}$ S. Chauhan, ${ }^{53}$ B. C. Choudhary, ${ }^{53}$ R. B. Garg, ${ }^{53}$ S. Keshri, ${ }^{53}$ A. Kumar, ${ }^{53}$ S. Malhotra, ${ }^{53}$ M. Naimuddin, ${ }^{53}$ K. Ranjan, ${ }^{53}$ R. Sharma, ${ }^{53}$ V. Sharma, ${ }^{53}$ R. Bhardwaj, ${ }^{54}$ R. Bhattacharya ${ }^{54}$ S. Bhattacharya,${ }^{54}$ U. Bhawandeep,${ }^{54}$ S. Dey, ${ }^{54}$ S. Dutt,${ }^{54}$ S. Dutta, ${ }^{54}$ S. Ghosh,${ }^{54}$ N. Majumdar,${ }^{54}$ A. Modak,${ }^{54}$ K. Mondal,${ }^{54}$ S. Mukhopadhyay,${ }^{54}$ S. Nandan,${ }^{54}$ A. Purohit, ${ }^{54}$ A. Roy, ${ }^{54}$ D. Roy, ${ }^{54}$ S. Roy Chowdhury ${ }^{54}$ S. Sarkar, ${ }^{54}$ M. Sharan, ${ }^{54}$ S. Thakur, ${ }^{54}$ P. K. Behera, ${ }^{55}$ R. Chudasama, ${ }^{56}$ D. Dutta ${ }^{56}$ V. Jha, ${ }^{56}$ V. Kumar, ${ }^{56}$ A. K. Mohanty, ${ }^{56,0}$ P. K. Netrakanti, ${ }^{56}$ L. M. Pant,${ }^{56}$ P. Shukla,${ }^{56}$ A. Topkar,${ }^{56}$ T. Aziz,${ }^{57}$ S. Dugad,${ }^{57}$ B. Mahakud,${ }^{57}$ S. Mitra,${ }^{57}$ G. B. Mohanty,${ }^{57}$ N. Sur, ${ }^{57}$ B. Sutar, ${ }^{57}$ S. Banerjee, ${ }^{58}$ S. Bhattacharya, ${ }^{58}$ S. Chatterjee, ${ }^{58}$ P. Das, ${ }^{58}$ M. Guchait,${ }^{58}$ Sa. Jain, ${ }^{58}$ S. Kumar, ${ }^{58}$ M. Maity, ${ }^{58, x}$ G. Majumder, ${ }^{58}$ K. Mazumdar,${ }^{58}$ T. Sarkar, ${ }^{58, x}$ N. Wickramage, ${ }^{58, y}$ S. Chauhan, ${ }^{59}$ S. Dube,${ }^{59}$ V. Hegde, ${ }^{59}$

A. Kapoor, ${ }^{59}$ K. Kothekar, ${ }^{59}$ S. Pandey, ${ }^{59}$ A. Rane, ${ }^{59}$ S. Sharma, ${ }^{59}$ S. Chenarani, ${ }^{60, z}$ E. Eskandari Tadavani, ${ }^{60}$ S. M. Etesami ${ }^{60, z}$ M. Khakzad,${ }^{60}$ M. Mohammadi Najafabadi, ${ }^{60}$ M. Naseri, ${ }^{60}$ S. Paktinat Mehdiabadi, ${ }^{60, a a}$

F. Rezaei Hosseinabadi, ${ }^{60}$ B. Safarzadeh, ${ }^{60, b b}$ M. Zeinali, ${ }^{60}$ M. Felcini, ${ }^{61}$ M. Grunewald, ${ }^{61}$ M. Abbrescia, ${ }^{62 a, 62 b}$

C. Calabria, ${ }^{62 a, 62 b}$ C. Caputo, ${ }^{62 a, 62 b}$ A. Colaleo, ${ }^{62 a}$ D. Creanza, ${ }^{62 a, 62 c}$ L. Cristella, ${ }^{62 a, 62 b}$ N. De Filippis ${ }^{62 a, 62 c}$

M. De Palma, ${ }^{62 a, 62 b}$ F. Errico, ${ }^{62 a, 62 b}$ L. Fiore, ${ }^{62 a}$ G. Iaselli, ${ }^{62 a, 62 c}$ S. Lezki, ${ }^{62 a, 62 b}$ G. Maggi, ${ }^{62 a, 62 c}$ M. Maggi, ${ }^{62 a}$

G. Miniello, ${ }^{62 a, 62 b}$ S. My, ${ }^{62 a, 62 b}$ S. Nuzzo,${ }^{62 a, 62 b}$ A. Pompili, ${ }^{62 a, 62 b}$ G. Pugliese, ${ }^{62 a, 62 c}$ R. Radogna, ${ }^{62 a, 62 b}$ A. Ranieri, ${ }^{62 a}$ G. Selvaggi, ${ }^{62 a, 62 b}$ A. Sharma, ${ }^{62 a}$ L. Silvestris, ${ }^{62 a, 0}$ R. Venditti, ${ }^{62 a}$ P. Verwilligen, ${ }^{62 a}$ G. Abbiendi, ${ }^{63 a}$ C. Battilana, ${ }^{63 a, 63 b}$ D. Bonacorsi, ${ }^{63 a, 63 b}$ S. Braibant-Giacomelli, ${ }^{63 a, 63 b}$ R. Campanini, ${ }^{63 a, 63 b}$ P. Capiluppi, ${ }^{63 a, 63 b}$ A. Castro, ${ }^{63 a, 63 b}$ F. R. Cavallo, ${ }^{63 a}$ S. S. Chhibra, ${ }^{63 a}$ G. Codispoti, ${ }^{63 a, 63 b}$ M. Cuffiani, ${ }^{63 a, 63 b}$ G. M. Dallavalle, ${ }^{63 a}$ F. Fabbri, ${ }^{63 a}$ A. Fanfani ${ }^{63 a, 63 b}$ D. Fasanella, ${ }^{63 a, 63 b}$ P. Giacomelli, ${ }^{63 a}$ C. Grandi, ${ }^{63 a}$ L. Guiducci, ${ }^{63 a, 63 b}$ S. Marcellini, ${ }^{63 a}$ G. Masetti, ${ }^{63 a}$ A. Montanari, ${ }^{63 a}$ F. L. Navarria, ${ }^{63 a, 63 b}$ A. Perrotta ${ }^{63 a}$ A. M. Rossi, ${ }^{63 a, 63 b}$ T. Rovelli, ${ }^{63 a, 63 b}$ G. P. Siroli, ${ }^{63 a, 63 b}$ N. Tosi, ${ }^{63 a}$ S. Albergo, ${ }^{64 a, 64 b}$ S. Costa, ${ }^{64 a, 64 b}$ A. Di Mattia, ${ }^{64 a}$ F. Giordano, ${ }^{64 a, 64 b}$ R. Potenza ${ }^{64 a, 64 b}$ A. Tricomi, ${ }^{64 a, 64 b}$ C. Tuve, ${ }^{64 a, 64 b}$ G. Barbagli, ${ }^{65 a}$ K. Chatterjee, ${ }^{65 a, 65 b}$ V. Ciulli, ${ }^{65 a, 65 b}$ C. Civinini, ${ }^{65 a}$ R. D’Alessandro, ${ }^{65 a, 65 b}$ E. Focardi ${ }^{65 a, 65 b}$ P. Lenzi, ${ }^{65 a, 65 b}$ M. Meschini ${ }^{65 a}$ S. Paoletti ${ }^{65 a}$ L. Russo, ${ }^{65 a, c c}$ G. Sguazzoni, ${ }^{65 a}$ D. Strom,${ }^{65 a}$ L. Viliani, ${ }^{65 a, 65 b, 0}$ L. Benussi, ${ }^{66}$ S. Bianco, ${ }^{66}$ F. Fabbri, ${ }^{66}$ D. Piccolo, ${ }^{66}$ F. Primavera, ${ }^{66,0}$ C. Biggio, ${ }^{67 a}$ V. Calvelli, ${ }^{67 a, 67 b}$ F. Ferro, ${ }^{67 a}$ E. Robutti, ${ }^{67 a}$ S. Tosi,${ }^{67 a, 67 b}$ L. Brianza, ${ }^{68 a, 68 b}$ F. Brivio, ${ }^{68 a, 68 b}$ V. Ciriolo, ${ }^{68 \mathrm{a}, 68 \mathrm{~b}}$ M. E. Dinardo, ${ }^{68 \mathrm{a}, 68 \mathrm{~b}}$ S. Fiorendi, ${ }^{68 \mathrm{a}, 68 \mathrm{~b}}$ S. Gennai ${ }^{68 \mathrm{a}}$ A. Ghezzi, ${ }^{68 \mathrm{a}, 68 \mathrm{~b}}$ P. Govoni, ${ }^{68 \mathrm{a}, 68 \mathrm{~b}}$ M. Malberti, ${ }^{68 \mathrm{a}, 68 \mathrm{~b}}$ S. Malvezzi, ${ }^{68 \mathrm{a}}$ R. A. Manzoni ${ }^{68 \mathrm{a}, 68 \mathrm{~b}}$ D. Menasce, ${ }^{68 \mathrm{a}}$ L. Moroni, ${ }^{68 \mathrm{a}}$ M. Paganoni, ${ }^{68 a, 68 \mathrm{~b}}$ K. Pauwels ${ }^{68 \mathrm{a}, 68 \mathrm{~b}}$ D. Pedrini, ${ }^{68 \mathrm{a}}$ S. Pigazzini, ${ }^{68 a, 68 b, d d}$ S. Ragazzi, ${ }^{68 a, 68 b}$ T. Tabarelli de Fatis, ${ }^{68 a, 68 b}$ S. Buontempo, ${ }^{69 a}$ N. Cavallo, ${ }^{69 a, 69 c}$ S. Di Guida, ${ }^{69 a, 69 d, 0}$ F. Fabozzi, ${ }^{69 a, 69 c}$ F. Fienga, ${ }^{69 a, 69 b}$ A. O. M. Iorio, ${ }^{69 a, 69 b}$ W. A. Khan, ${ }^{69 a}$ L. Lista, ${ }^{69 a}$ S. Meola, ${ }^{69 a, 69 d, o}$ P. Paolucci, ${ }^{69 a, o}$ C. Sciacca ${ }^{69 a, 69 b}$ F. Thyssen, ${ }^{69 a}$ P. Azzi, ${ }^{70 a, 0}$ N. Bacchetta, ${ }^{70 a}$ L. Benato, ${ }^{70 a, 70 b}$ D. Bisello, ${ }^{70 a, 70 b}$ A. Boletti, ${ }^{70 a, 70 b}$ R. Carlin, ${ }^{70 \mathrm{a}, 70 \mathrm{~b}}$ A. Carvalho Antunes De Oliveira, ${ }^{70 a, 70 b}$ P. Checchia, ${ }^{70 \mathrm{a}}$ P. De Castro Manzano, ${ }^{70 \mathrm{a}}$ T. Dorigo, ${ }^{70 \mathrm{a}}$ F. Gasparini, ${ }^{70 a, 70 b}$ U. Gasparini, ${ }^{70 a, 70 b}$ A. Gozzelino, ${ }^{70 a}$ S. Lacaprara, ${ }^{70 a}$ P. Lujan, ${ }^{70 a}$ M. Margoni, ${ }^{70 a, 70 b}$ 
A. T. Meneguzzo, ${ }^{70 a, 70 b}$ N. Pozzobon, ${ }^{70 a, 70 b}$ P. Ronchese,${ }^{70 a, 70 b}$ R. Rossin,${ }^{70 a, 70 b}$ F. Simonetto, ${ }^{70 a, 70 b}$ E. Torassa, ${ }^{70 a}$ M. Zanetti, ${ }^{70 a, 70 b}$ P. Zotto, ${ }^{70 a, 70 b}$ G. Zumerle, ${ }^{70 a, 70 b}$ A. Braghieri, ${ }^{71 a}$ F. Fallavollita, ${ }^{71 a, 71 b}$ A. Magnani,${ }^{71 a, 71 b}$ P. Montagna,${ }^{71 a, 71 b}$ S. P. Ratti, ${ }^{71 a, 71 b}$ V. Re, ${ }^{71 a}$ M. Ressegotti, ${ }^{71 a}$ C. Riccardi, ${ }^{71 a, 71 b}$ P. Salvini, ${ }^{71 a}$ I. Vai, ${ }^{71 a, 71 b}$ P. Vitulo, ${ }^{71 a, 71 b}$

L. Alunni Solestizi, ${ }^{72 a, 72 b}$ M. Biasini, ${ }^{72 a, 72 b}$ G. M. Bilei, ${ }^{72 a}$ C. Cecchi, ${ }^{72 a, 72 b}$ D. Ciangottini, ${ }^{72 a, 72 b}$ L. Fanò, ${ }^{72 a, 72 b}$ P. Lariccia, ${ }^{72 a, 72 b}$ R. Leonardi, ${ }^{72 a, 72 b}$ E. Manoni, ${ }^{72 a}$ G. Mantovani, ${ }^{72 a, 72 b}$ V. Mariani, ${ }^{72 a, 72 b}$ M. Menichelli, ${ }^{72 a}$ A. Rossi, ${ }^{72 a, 72 b}$ A. Santocchia ${ }^{72 a, 72 b}$ D. Spiga, ${ }^{72 a}$ K. Androsov ${ }^{73 a}$ P. Azzurri,${ }^{73 a, o}$ G. Bagliesi, ${ }^{73 a}$ J. Bernardini, ${ }^{73 a}$ T. Boccali, ${ }^{73 a}$ L. Borrello, ${ }^{73 a}$ R. Castaldi, ${ }^{73 a}$ M. A. Ciocci ${ }^{73 a, 73 b}$ R. Dell'Orso, ${ }^{73 a}$ G. Fedi, ${ }^{73 a}$ L. Giannini, ${ }^{73 a, 73 c}$ A. Giassi, ${ }^{73 a}$ M. T. Grippo, ${ }^{73 a, c c}$ F. Ligabue, ${ }^{73 a, 73 c}$ T. Lomtadze, ${ }^{73 a}$ E. Manca, ${ }^{73 a, 73 c}$ G. Mandorli, ${ }^{73 a, 73 c}$ L. Martini, ${ }^{73 a, 73 b}$ A. Messineo, ${ }^{73 a, 73 b}$ F. Palla, ${ }^{73 a}$ A. Rizzi, ${ }^{73 a, 73 b}$ A. Savoy-Navarro, ${ }^{73 a, e e}$ P. Spagnolo, ${ }^{73 a}$ R. Tenchini,${ }^{73 a}$ G. Tonelli, ${ }^{73 a, 73 b}$ A. Venturi, ${ }^{73 a}$ P. G. Verdini, ${ }^{73 a}$ L. Barone,${ }^{74 a 7 b}$ F. Cavallari, ${ }^{74 a}$ M. Cipriani, ${ }^{74 a, 74 b}$ N. Daci,${ }^{74 a}$ D. Del Re,${ }^{74 a, 74 b, o}$ M. Diemoz, ${ }^{74 a}$ S. Gelli, ${ }^{74 a, 74 b}$ E. Longo, ${ }^{74 a, 74 b}$ F. Margaroli, ${ }^{74 a, 74 b}$ B. Marzocchi, ${ }^{74 a, 74 b}$ P. Meridiani, ${ }^{74 a}$ G. Organtini, ${ }^{74 a, 74 b}$ R. Paramatti, ${ }^{74 a, 74 b}$

F. Preiato, ${ }^{74 a, 74 b}$ S. Rahatlou, ${ }^{74 a, 74 b}$ C. Rovelli, ${ }^{74 a}$ F. Santanastasio, ${ }^{74 a, 74 b}$ N. Amapane, ${ }^{75 a, 75 b}$ R. Arcidiacono, ${ }^{75 a, 75 c}$

S. Argiro, ${ }^{75 a, 75 b}$ M. Arneodo, ${ }^{75 a, 75 c}$ N. Bartosik ${ }^{75 a}$ R. Bellan, ${ }^{75 a, 75 b}$ C. Biino, ${ }^{75 a}$ N. Cartiglia, ${ }^{75 a}$ F. Cenna, ${ }^{75 a, 75 b}$

M. Costa,${ }^{75 a, 75 b}$ R. Covarellii, ${ }^{75 a, 75 b}$ A. Degano,${ }^{75 a, 75 b}$ N. Demaria, ${ }^{75 a}$ B. Kiani, ${ }^{75 a, 75 b}$ C. Mariotti, ${ }^{75 a}$ S. Maselli, ${ }^{75 a}$ E. Migliore, ${ }^{75 a, 75 b}$ V. Monaco, ${ }^{75 a, 75 b}$ E. Monteil,${ }^{75 a, 75 b}$ M. Monteno, ${ }^{75 a}$ M. M. Obertino, ${ }^{75 a, 75 b}$ L. Pacher, ${ }^{75 a, 75 b}$ N. Pastrone,${ }^{75 a}$ M. Pelliccioni, ${ }^{75 a}$ G. L. Pinna Angioni ${ }^{75 a, 75 b}$ F. Ravera ${ }^{75 a, 75 b}$ A. Romero, ${ }^{75 a, 75 b}$ M. Ruspa ${ }^{75 a, 75 c}$ R. Sacchi, ${ }^{75 a, 75 b}$ K. Shchelina, ${ }^{75 a, 75 b}$ V. Sola,${ }^{75 a}$ A. Solano, ${ }^{75 a, 75 b}$ A. Staiano, ${ }^{75 a}$ P. Traczyk, ${ }^{75 a, 75 b}$ S. Belforte, ${ }^{76 a}$ M. Casarsa,${ }^{76 a}$ F. Cossutti, ${ }^{76 a}$ G. Della Ricca, ${ }^{76 a, 76 b}$ A. Zanetti, ${ }^{76 a}$ D. H. Kim, ${ }^{77}$ G. N. Kim ${ }^{77}$ M. S. Kim, ${ }^{77}$ J. Lee,${ }^{77}$ S. Lee, ${ }^{77}$ S. W. Lee, ${ }^{77}$ C. S. Moon, ${ }^{77}$ Y. D. Oh, ${ }^{77}$ S. Sekmen, ${ }^{77}$ D. C. Son, ${ }^{77}$ Y. C. Yang, ${ }^{77}$ A. Lee, ${ }^{78}$ H. Kim, ${ }^{79}$ D. H. Moon, ${ }^{79}$ G. Oh, ${ }^{79}$ J. A. Brochero Cifuentes,${ }^{80}$ J. Goh ${ }^{80}$ T. J. Kim, ${ }^{80}$ S. Cho, ${ }^{81}$ S. Choi, ${ }^{81}$ Y. Go, ${ }^{81}$ D. Gyun, ${ }^{81}$ S. Ha, ${ }^{81}$ B. Hong,${ }^{81}$ Y. Jo, ${ }^{81}$ Y. Kim, ${ }^{81}$ K. Lee, ${ }^{81}$ K. S. Lee, ${ }^{81}$ S. Lee, ${ }^{81}$ J. Lim, ${ }^{81}$ S. K. Park, ${ }^{81}$ Y. Roh, ${ }^{81}$ J. Almond, ${ }^{82}$ J. Kim, ${ }^{82}$ J. S. Kim ${ }^{82}$ H. Lee, ${ }^{82}$ K. Lee, ${ }^{82}$ K. Nam, ${ }^{82}$ S. B. Oh, ${ }^{82}$ B. C. Radburn-Smith, ${ }^{82}$ S. h. Seo ${ }^{82}$ U. K. Yang, ${ }^{82}$ H. D. Yoo, ${ }^{82}$ G. B. Yu, ${ }^{82}$ M. Choi ${ }^{83}$ H. Kim, ${ }^{83}$ J. H. Kim,${ }^{83}$ J. S. H. Lee, ${ }^{83}$ I. C. Park, ${ }^{83}$ G. Ryu ${ }^{83}$ Y. Choi, ${ }^{84}$ C. Hwang, ${ }^{84}$ J. Lee, ${ }^{84}$ I. Yu ${ }^{84}$ V. Dudenas, ${ }^{85}$ A. Juodagalvis, ${ }^{85}$ J. Vaitkus, ${ }^{85}$ I. Ahmed ${ }^{86}$ Z. A. Ibrahim, ${ }^{86}$ M. A. B. Md Ali, ${ }^{86, f f}$ F. Mohamad Idris, ${ }^{86, g g}$ W. A. T. Wan Abdullah, ${ }^{86}$ M. N. Yusli, ${ }^{86}$ Z. Zolkapli, ${ }^{86}$ R. Reyes-Almanza, ${ }^{87}$ G. Ramirez-Sanchez, ${ }^{87}$ M. C. Duran-Osuna, ${ }^{87}$ H. Castilla-Valdez, ${ }^{87}$ E. De La Cruz-Burelo, ${ }^{87}$ I. Heredia-De La Cruz, ${ }^{87, h h}$ R. I. Rabadan-Trejo, ${ }^{87}$ R. Lopez-Fernandez, ${ }^{87}$ J. Mejia Guisao, ${ }^{87}$ A. Sanchez-Hernandez, ${ }^{87}$ S. Carrillo Moreno, ${ }^{88}$ C. Oropeza Barrera, ${ }^{88}$ F. Vazquez Valencia, ${ }^{88}$ I. Pedraza, ${ }^{89}$ H. A. Salazar Ibarguen, ${ }^{89}$ C. Uribe Estrada, ${ }^{89}$ A. Morelos Pineda, ${ }^{90}$ D. Krofcheck, ${ }^{91}$ P. H. Butler, ${ }^{92}$ A. Ahmad, ${ }^{93}$ M. Ahmad, ${ }^{93}$ Q. Hassan, ${ }^{93}$ H. R. Hoorani, ${ }^{93}$ A. Saddique, ${ }^{93}$ M. A. Shah, ${ }^{93}$ M. Shoaib,${ }^{93}$ M. Waqas, ${ }^{93}$ H. Bialkowska, ${ }^{94}$ M. Bluj,${ }^{94}$ B. Boimska, ${ }^{94}$ T. Frueboes,${ }^{94}$ M. Górski, ${ }^{94}$ M. Kazana, ${ }^{94}$ K. Nawrocki, ${ }^{94}$ K. Romanowska-Rybinska, ${ }^{94}$ M. Szleper, ${ }^{94}$ P. Zalewski, ${ }^{94}$ K. Bunkowski, ${ }^{95}$ A. Byszuk, ${ }^{95, i i}$ K. Doroba, ${ }^{95}$ A. Kalinowski, ${ }^{95}$ M. Konecki, ${ }^{95}$ J. Krolikowski, ${ }^{95}$ M. Misiura, ${ }^{95}$ M. Olszewski, ${ }^{95}$ A. Pyskir, ${ }^{95}$ M. Walczak, ${ }^{95}$ P. Bargassa, ${ }^{96}$ C. Beirão Da Cruz E Silva, ${ }^{96}$ B. Calpas, ${ }^{96, j j}$ A. Di Francesco, ${ }^{96}$ P. Faccioli, ${ }^{96}$ M. Gallinaro, ${ }^{96}$ J. Hollar,${ }^{96}$ N. Leonardo, ${ }^{96}$ L. Lloret Iglesias, ${ }^{96}$ M. V. Nemallapudi, ${ }^{96}$ J. Seixas,${ }^{96}$ O. Toldaiev,${ }^{96}$ D. Vadruccio ${ }^{96}$ J. Varela,${ }^{96}$ S. Afanasiev,${ }^{97}$ P. Bunin, ${ }^{97}$ M. Gavrilenko, ${ }^{97}$ I. Golutvin, ${ }^{97}$ I. Gorbunov, ${ }^{97}$ A. Kamenev, ${ }^{97}$ V. Karjavin, ${ }^{97}$ A. Lanev, ${ }^{97}$ A. Malakhov, ${ }^{97}$ V. Matveev, ${ }^{97, k k, 11}$ V. Palichik,${ }^{97}$ V. Perelygin, ${ }^{97}$ S. Shmatov, ${ }^{97}$ S. Shulha,${ }^{97}$ N. Skatchkov, ${ }^{97}$ V. Smirnov, ${ }^{97}$ N. Voytishin, ${ }^{97}$ A. Zarubin, ${ }^{97}$ Y. Ivanov, ${ }^{98}$ V. Kim, ${ }^{98, m m}$ E. Kuznetsova, ${ }^{98, n n}$ P. Levchenko, ${ }^{98}$ V. Murzin, ${ }^{98}$ V. Oreshkin, ${ }^{98}$ I. Smirnov, ${ }^{98}$ V. Sulimov, ${ }^{98}$ L. Uvarov, ${ }^{98}$ S. Vavilov, ${ }^{98}$ A. Vorobyev, ${ }^{98}$ Yu. Andreev, ${ }^{99}$ A. Dermenev, ${ }^{99}$ S. Gninenko, ${ }^{99}$ N. Golubev, ${ }^{99}$ A. Karneyeu ${ }^{99}$ M. Kirsanov, ${ }^{99}$ N. Krasnikov, ${ }^{99}$ A. Pashenkov, ${ }^{99}$ D. Tlisov, ${ }^{99}$

A. Toropin, ${ }^{99}$ V. Epshteyn, ${ }^{100}$ V. Gavrilov, ${ }^{100}$ N. Lychkovskaya, ${ }^{100}$ V. Popov, ${ }^{100}$ I. Pozdnyakov, ${ }^{100}$ G. Safronov, ${ }^{100}$ A. Spiridonov, ${ }^{100}$ A. Stepennov, ${ }^{100}$ M. Toms, ${ }^{100}$ E. Vlasov, ${ }^{100}$ A. Zhokin, ${ }^{100}$ T. Aushev, ${ }^{101}$ A. Bylinkin, ${ }^{101,11}$ R. Chistov, ${ }^{102,00}$ M. Danilov, ${ }^{102,00}$ P. Parygin, ${ }^{102}$ D. Philippov, ${ }^{102}$ S. Polikarpov, ${ }^{102}$ E. Tarkovskii, ${ }^{102}$ V. Andreev, ${ }^{103}$ M. Azarkin, ${ }^{103,11}$ I. Dremin, ${ }^{103,11}$ M. Kirakosyan, ${ }^{103,11}$ A. Terkulov, ${ }^{103}$ A. Baskakov, ${ }^{104}$ A. Belyaev, ${ }^{104}$ E. Boos, ${ }^{104}$ M. Dubinin, ${ }^{104, p p}$ L. Dudko, ${ }^{104}$ A. Ershov, ${ }^{104}$ A. Gribushin, ${ }^{104}$ V. Klyukhin, ${ }^{104}$ O. Kodolova, ${ }^{104}$ I. Lokhtin, ${ }^{104}$ I. Miagkov, ${ }^{104}$ S. Obraztsov, ${ }^{104}$ S. Petrushanko, ${ }^{104}$ V. Savrin, ${ }^{104}$ A. Snigirev, ${ }^{104}$ V. Blinov, ${ }^{105, \mathrm{qq}}$ Y. Skovpen, ${ }^{105, \mathrm{qq}}$ D. Shtol, ${ }^{105, \mathrm{qq}}$ I. Azhgirey ${ }^{106}$ I. Bayshev, ${ }^{106}$ S. Bitioukov, ${ }^{106}$ D. Elumakhov, ${ }^{106}$ V. Kachanov, ${ }^{106}$ A. Kalinin, ${ }^{106}$ D. Konstantinov, ${ }^{106}$ V. Krychkine, ${ }^{106}$ V. Petrov, ${ }^{106}$

R. Ryutin, ${ }^{106}$ A. Sobol,${ }^{106}$ S. Troshin, ${ }^{106}$ N. Tyurin, ${ }^{106}$ A. Uzunian, ${ }^{106}$ A. Volkov, ${ }^{106}$ P. Adzic, ${ }^{107, r r}$ P. Cirkovic, ${ }^{107}$

D. Devetak, ${ }^{107}$ M. Dordevic, ${ }^{107}$ J. Milosevic, ${ }^{107}$ V. Rekovic,${ }^{107}$ J. Alcaraz Maestre, ${ }^{108}$ M. Barrio Luna,${ }^{108}$ M. Cerrada, ${ }^{108}$

N. Colino, ${ }^{108}$ B. De La Cruz, ${ }^{108}$ A. Delgado Peris, ${ }^{108}$ A. Escalante Del Valle, ${ }^{108}$ C. Fernandez Bedoya,${ }^{108}$

J. P. Fernández Ramos, ${ }^{108}$ J. Flix, ${ }^{108}$ M. C. Fouz, ${ }^{108}$ P. Garcia-Abia, ${ }^{108}$ O. Gonzalez Lopez, ${ }^{108}$ S. Goy Lopez, ${ }^{108}$ 
J. M. Hernandez, ${ }^{108}$ M. I. Josa, ${ }^{108}$ A. Pérez-Calero Yzquierdo, ${ }^{108}$ J. Puerta Pelayo, ${ }^{108}$ A. Quintario Olmeda, ${ }^{108}$ I. Redondo, ${ }^{108}$ L. Romero, ${ }^{108}$ M. S. Soares, ${ }^{108}$ A. Álvarez Fernández, ${ }^{108}$ J. F. de Trocóniz, ${ }^{109}$ M. Missiroli, ${ }^{109}$ D. Moran, ${ }^{109}$ J. Cuevas, ${ }^{110}$ C. Erice, ${ }^{110}$ J. Fernandez Menendez, ${ }^{110}$ I. Gonzalez Caballero, ${ }^{110}$ J. R. González Fernández, ${ }^{110}$

E. Palencia Cortezon, ${ }^{110}$ S. Sanchez Cruz, ${ }^{110}$ I. Suárez Andrés, ${ }^{110}$ P. Vischia,${ }^{110}$ J. M. Vizan Garcia, ${ }^{110}$ I. J. Cabrillo, ${ }^{111}$ A. Calderon, ${ }^{111}$ B. Chazin Quero, ${ }^{111}$ E. Curras, ${ }^{111}$ J. Duarte Campderros, ${ }^{111}$ M. Fernandez, ${ }^{111}$ J. Garcia-Ferrero, ${ }^{111}$

G. Gomez ${ }^{111}$ A. Lopez Virto, ${ }^{111}$ J. Marco,${ }^{111}$ C. Martinez Rivero, ${ }^{111}$ P. Martinez Ruiz del Arbol, ${ }^{111}$ F. Matorras, ${ }^{111}$ J. Piedra Gomez, ${ }^{111}$ T. Rodrigo, ${ }^{111}$ A. Ruiz-Jimeno, ${ }^{111}$ L. Scodellaro, ${ }^{111}$ N. Trevisani,${ }^{111}$ I. Vila, ${ }^{111}$ R. Vilar Cortabitarte, ${ }^{111}$ D. Abbaneo, ${ }^{112}$ E. Auffray, ${ }^{112}$ P. Baillon, ${ }^{12}$ A. H. Ball, ${ }^{112}$ D. Barney, ${ }^{112}$ M. Bianco, ${ }^{112}$ P. Bloch, ${ }^{112}$ A. Bocci, ${ }^{112}$ C. Botta, ${ }^{112}$ T. Camporesi, ${ }^{112}$ R. Castello, ${ }^{112}$ M. Cepeda, ${ }^{112}$ G. Cerminara, ${ }^{112}$ E. Chapon, ${ }^{112}$ Y. Chen, ${ }^{112}$ D. d'Enterria, ${ }^{112}$

A. Dabrowski, ${ }^{112}$ V. Daponte, ${ }^{112}$ A. David, ${ }^{112}$ M. De Gruttola, ${ }^{112}$ A. De Roeck, ${ }^{112}$ E. Di Marco, ${ }^{112, s s}$ M. Dobson, ${ }^{112}$ B. Dorney, ${ }^{112}$ T. du Pree, ${ }^{112}$ M. Dünser, ${ }^{112}$ N. Dupont, ${ }^{112}$ A. Elliott-Peisert, ${ }^{112}$ P. Everaerts, ${ }^{112}$ G. Franzoni,${ }^{112}$ J. Fulcher, ${ }^{112}$ W. Funk ${ }^{112}$ D. Gigi, ${ }^{112}$ K. Gill, ${ }^{112}$ F. Glege, ${ }^{112}$ D. Gulhan, ${ }^{112}$ S. Gundacker, ${ }^{112}$ M. Guthoff, ${ }^{112}$ P. Harris, ${ }^{112}$ J. Hegeman, ${ }^{112}$ V. Innocente, ${ }^{112}$ P. Janot, ${ }^{112}$ O. Karacheban, ${ }^{112, \mathrm{r}}$ J. Kieseler, ${ }^{112}$ H. Kirschenmann, ${ }^{112}$ V. Knünz, ${ }^{112}$ A. Kornmayer, ${ }^{12,0}$ M. J. Kortelainen, ${ }^{112}$ M. Krammer, ${ }^{112, b}$ C. Lange, ${ }^{112}$ P. Lecoq ${ }^{112}$ C. Lourenço, ${ }^{112}$ M. T. Lucchini, ${ }^{112}$ L. Malgeri, ${ }^{112}$ M. Mannelli, ${ }^{112}$ A. Martelli, ${ }^{112}$ F. Meijers, ${ }^{112}$ J. A. Merlin, ${ }^{112}$ S. Mersi, ${ }^{112}$ E. Meschi, ${ }^{112}$ P. Milenovic,${ }^{112, t t}$ F. Moortgat, ${ }^{112}$ M. Mulders, ${ }^{112}$ H. Neugebauer, ${ }^{112}$ S. Orfanelli, ${ }^{112}$ L. Orsini,${ }^{112}$ L. Pape, ${ }^{112}$ E. Perez,${ }^{112}$ M. Peruzzi, ${ }^{112}$ A. Petrilli, ${ }^{112}$ G. Petrucciani, ${ }^{112}$ A. Pfeiffer, ${ }^{112}$ M. Pierini, ${ }^{112}$ A. Racz, ${ }^{112}$ T. Reis, ${ }^{112}$ G. Rolandi, ${ }^{112, \text { uu }}$ M. Rovere,${ }^{112}$ H. Sakulin, ${ }^{112}$ C. Schäfer, ${ }^{112}$ C. Schwick, ${ }^{112}$ M. Seidel,${ }^{112}$ M. Selvaggi, ${ }^{112}$ A. Sharma, ${ }^{112}$ P. Silva ${ }^{112}$ P. Sphicas, ${ }^{12, \text { vv }}$ A. Stakia, ${ }^{112}$ J. Steggemann, ${ }^{112}$ M. Stoye, ${ }^{112}$ M. Tosi, ${ }^{112}$ D. Treille, ${ }^{112}$ A. Triossi, ${ }^{112}$ A. Tsirou,${ }^{112}$ V. Veckalns, ${ }^{112, w w}$ G. I. Veres, ${ }^{112, t}$ M. Verweij, ${ }^{112}$ N. Wardle, ${ }^{112}$ W. D. Zeuner, ${ }^{112}$ W. Bertl, ${ }^{113, a}$ L. Caminada,,${ }^{11, x x}$ K. Deiters, ${ }^{113}$ W. Erdmann, ${ }^{113}$ R. Horisberger ${ }^{113}$ Q. Ingram, ${ }^{113}$ H. C. Kaestli, ${ }^{113}$ D. Kotlinski, ${ }^{113}$ U. Langenegger, ${ }^{113}$ T. Rohe, ${ }^{113}$ S. A. Wiederkehr, ${ }^{113}$ F. Bachmair, ${ }^{114}$ L. Bäni,${ }^{114}$ P. Berger,${ }^{114}$ L. Bianchini, ${ }^{114}$ B. Casal, ${ }^{114}$ G. Dissertori, ${ }^{114}$ M. Dittmar, ${ }^{114}$ M. Donegà,${ }^{114}$ C. Grab, ${ }^{114}$ C. Heidegger, ${ }^{114}$ D. Hits, ${ }^{114}$ J. Hoss, ${ }^{114}$ G. Kasieczka, ${ }^{114}$ T. Klijnsma, ${ }^{114}$ W. Lustermann, ${ }^{114}$ B. Mangano, ${ }^{114}$ M. Marionneau, ${ }^{114}$ M. T. Meinhard, ${ }^{114}$ D. Meister, ${ }^{114}$ F. Micheli, ${ }^{114}$ P. Musella, ${ }^{114}$ F. Nessi-Tedaldi, ${ }^{114}$ F. Pandolfi, ${ }^{114}$ J. Pata, ${ }^{114}$ F. Pauss, ${ }^{114}$ G. Perrin, ${ }^{114}$ L. Perrozzi, ${ }^{114}$ M. Quittnat, ${ }^{114}$ M. Reichmann, ${ }^{114}$ M. Schönenberger, ${ }^{114}$ L. Shchutska, ${ }^{114}$ V. R. Tavolaro, ${ }^{114}$ K. Theofilatos, ${ }^{114}$ M. L. Vesterbacka Olsson, ${ }^{114}$ R. Wallny, ${ }^{114}$ D. H. Zhu, ${ }^{114}$ T. K. Aarrestad, ${ }^{115}$ C. Amsler, ${ }^{115, y y}$ M. F. Canelli, ${ }^{115}$ A. De Cosa,${ }^{115}$ R. Del Burgo,${ }^{115}$ S. Donato,${ }^{115}$ C. Galloni, ${ }^{115}$ T. Hreus, ${ }^{115}$ B. Kilminster, ${ }^{115}$ J. Ngadiuba, ${ }^{115}$ D. Pinna, ${ }^{115}$ G. Rauco, ${ }^{115}$ P. Robmann, ${ }^{115}$ D. Salerno, ${ }^{115}$ C. Seitz, ${ }^{115}$ Y. Takahashi, ${ }^{115}$ A. Zucchetta, ${ }^{115}$ V. Candelise, ${ }^{116}$ T. H. Doan, ${ }^{116}$ Sh. Jain, ${ }^{116}$ R. Khurana, ${ }^{116}$ C. M. Kuo, ${ }^{116}$ W. Lin, ${ }^{116}$ A. Pozdnyakov, ${ }^{116}$ S. S. Yu, ${ }^{116}$ Arun Kumar, ${ }^{117}$ P. Chang, ${ }^{117}$ Y. Chao, ${ }^{117}$ K. F. Chen, ${ }^{117}$ P. H. Chen, ${ }^{117}$ F. Fiori, ${ }^{117}$ W.-S. Hou, ${ }^{117}$ Y. Hsiung, ${ }^{117}$ Y. F. Liu, ${ }^{117}$ R.-S. Lu, ${ }^{117}$ E. Paganis, ${ }^{117}$ A. Psallidas, ${ }^{117}$ A. Steen, ${ }^{117}$ J. f. Tsai, ${ }^{117}$ B. Asavapibhop, ${ }^{118}$ K. Kovitanggoon, ${ }^{118}$ G. Singh, ${ }^{118}$ N. Srimanobhas, ${ }^{118}$ A. Adiguzel, ${ }^{119, z z}$ F. Boran, ${ }^{119}$ S. Cerci, ${ }^{119, \text { aaa }}$ S. Damarseckin, ${ }^{119}$ Z. S. Demiroglu, ${ }^{119}$ C. Dozen, ${ }^{119}$ I. Dumanoglu, ${ }^{119}$ S. Girgis, ${ }^{119}$ G. Gokbulut, ${ }^{119}$ Y. Guler, ${ }^{119}$ I. Hos, ${ }^{119, b b b}$ E. E. Kangal, ${ }^{11, \text {,cc }}$ O. Kara, ${ }^{119}$ A. Kayis Topaksu, ${ }^{119}$ U. Kiminsu, ${ }^{119}$ M. Oglakci, ${ }^{119}$ G. Onengut, ${ }^{119, \text { ddd }}$ K. Ozdemir, ${ }^{119, \text { eee }}$ D. Sunar Cerci, ${ }^{119, \text { aaa }}$ B. Tali, ${ }^{19, \text { aaa }}$ S. Turkcapar, ${ }^{119}$ I. S. Zorbakir, ${ }^{119}$ C. Zorbilmez, ${ }^{119}$ B. Bilin, ${ }^{120}$ G. Karapinar, ${ }^{120, f f f}$ K. Ocalan, ${ }^{120, g g g}$ M. Yalvac, ${ }^{120}$ M. Zeyrek, ${ }^{120}$ E. Gülmez, ${ }^{121}$ M. Kaya, ${ }^{121, \text { hhh }}$ O. Kaya, ${ }^{121, \text { iii }}$ S. Tekten, ${ }^{121}$ E. A. Yetkin, ${ }^{121, j j j}$ M. N. Agaras, ${ }^{122}$ S. Atay, ${ }^{122}$ A. Cakir, ${ }^{122}$ K. Cankocak, ${ }^{122}$ B. Grynyov, ${ }^{123}$ L. Levchuk, ${ }^{124}$ P. Sorokin, ${ }^{124}$ R. Aggleton, ${ }^{125}$ F. Ball, ${ }^{125}$ L. Beck, ${ }^{125}$ J. J. Brooke, ${ }^{125}$ D. Burns, ${ }^{125}$ E. Clement, ${ }^{125}$ D. Cussans, ${ }^{125}$ O. Davignon, ${ }^{125}$ H. Flacher, ${ }^{125}$ J. Goldstein, ${ }^{125}$ M. Grimes, ${ }^{125}$ G. P. Heath, ${ }^{125}$ H. F. Heath, ${ }^{125}$ J. Jacob, ${ }^{125}$ L. Kreczko, ${ }^{125}$ C. Lucas, ${ }^{125}$ D. M. Newbold, ${ }^{125, k k k}$ S. Paramesvaran, ${ }^{125}$ A. Poll, ${ }^{125}$ T. Sakuma, ${ }^{125}$ S. Seif El Nasr-storey, ${ }^{125}$ D. Smith, ${ }^{125}$ V. J. Smith, ${ }^{125}$ K. W. Bell, ${ }^{126}$ A. Belyaev, ${ }^{126,111}$ C. Brew, ${ }^{126}$ R. M. Brown, ${ }^{126}$ L. Calligaris, ${ }^{126}$ D. Cieri, ${ }^{126}$ D. J. A. Cockerill, ${ }^{126}$ J. A. Coughlan, ${ }^{126}$ K. Harder, ${ }^{126}$ S. Harper, ${ }^{126}$ E. Olaiya,${ }^{126}$ D. Petyt, ${ }^{126}$ C. H. Shepherd-Themistocleous, ${ }^{126}$ A. Thea, ${ }^{126}$ I. R. Tomalin, ${ }^{126}$ T. Williams, ${ }^{126}$ G. Auzinger, ${ }^{127}$ R. Bainbridge, ${ }^{127}$ S. Breeze, ${ }^{127}$ O. Buchmuller, ${ }^{127}$ A. Bundock, ${ }^{127}$ S. Casasso,${ }^{127}$ M. Citron, ${ }^{127}$ D. Colling, ${ }^{127}$ L. Corpe,${ }^{127}$ P. Dauncey, ${ }^{127}$ G. Davies ${ }^{127}$ A. De Wit, ${ }^{127}$ M. Della Negra, ${ }^{127}$ R. Di Maria, ${ }^{127}$ A. Elwood, ${ }^{127}$ Y. Haddad, ${ }^{127}$ G. Hall, ${ }^{127}$ G. Iles, ${ }^{127}$ T. James, ${ }^{127}$ R. Lane, ${ }^{127}$ C. Laner, ${ }^{127}$ L. Lyons, ${ }^{127}$ A.-M. Magnan, ${ }^{127}$ S. Malik, ${ }^{127}$ L. Mastrolorenzo, ${ }^{127}$ T. Matsushita, ${ }^{127}$ J. Nash, ${ }^{127}$ A. Nikitenko, ${ }^{127, g}$ V. Palladino, ${ }^{127}$ M. Pesaresi, ${ }^{127}$ D. M. Raymond, ${ }^{127}$ A. Richards,${ }^{127}$ A. Rose,${ }^{127}$ E. Scott, ${ }^{127}$ C. Seez ${ }^{127}$ A. Shtipliyski, ${ }^{127}$ S. Summers, ${ }^{127}$ A. Tapper, ${ }^{127}$ K. Uchida, ${ }^{127}$ M. Vazquez Acosta, ${ }^{127, m m m}$ T. Virdee, ${ }^{127,0}$ D. Winterbottom, ${ }^{127}$ J. Wright, ${ }^{127}$ S. C. Zenz, ${ }^{127}$ J. E. Cole, ${ }^{128}$ P. R. Hobson, ${ }^{128}$ A. Khan, ${ }^{128}$ P. Kyberd, ${ }^{128}$ I. D. Reid, ${ }^{128}$ P. Symonds, ${ }^{128}$ L. Teodorescu, ${ }^{128}$ M. Turner, ${ }^{128}$ A. Borzou, ${ }^{129}$ K. Call, ${ }^{129}$ J. Dittmann, ${ }^{129}$ K. Hatakeyama, ${ }^{129}$ H. Liu, ${ }^{129}$ 
N. Pastika, ${ }^{129}$ C. Smith, ${ }^{129}$ R. Bartek, ${ }^{130}$ A. Dominguez, ${ }^{130}$ A. Buccilli, ${ }^{131}$ S. I. Cooper, ${ }^{131}$ C. Henderson, ${ }^{131}$ P. Rumerio, ${ }^{131}$ C. West,${ }^{131}$ D. Arcaro, ${ }^{132}$ A. Avetisyan, ${ }^{132}$ T. Bose, ${ }^{132}$ D. Gastler, ${ }^{132}$ D. Rankin, ${ }^{132}$ C. Richardson, ${ }^{132}$ J. Rohlf, ${ }^{132}$ L. Sulak, ${ }^{132}$ D. Zou, ${ }^{132}$ G. Benelli, ${ }^{133}$ D. Cutts, ${ }^{133}$ A. Garabedian, ${ }^{133}$ J. Hakala, ${ }^{133}$ U. Heintz, ${ }^{133}$ J. M. Hogan, ${ }^{133}$ K. H. M. Kwok, ${ }^{133}$ E. Laird, ${ }^{133}$ G. Landsberg, ${ }^{133}$ Z. Mao, ${ }^{133}$ M. Narain, ${ }^{133}$ J. Pazzini, ${ }^{133}$ S. Piperov, ${ }^{133}$ S. Sagir, ${ }^{133}$ R. Syarif, ${ }^{133}$ D. Yu, ${ }^{133}$ R. Band, ${ }^{134}$ C. Brainerd, ${ }^{134}$ D. Burns, ${ }^{134}$ M. Calderon De La Barca Sanchez, ${ }^{134}$ M. Chertok, ${ }^{134}$ J. Conway, ${ }^{134}$ R. Conway, ${ }^{134}$ P. T. Cox,${ }^{134}$ R. Erbacher, ${ }^{134}$ C. Flores,${ }^{134}$ G. Funk,${ }^{134}$ M. Gardner, ${ }^{134}$ W. Ko, ${ }^{134}$ R. Lander, ${ }^{134}$ C. Mclean,${ }^{134}$ M. Mulhearn, ${ }^{134}$ D. Pellett, ${ }^{134}$ J. Pilot, ${ }^{134}$ S. Shalhout, ${ }^{134}$ M. Shi, ${ }^{134}$ J. Smith,${ }^{134}$ M. Squires, ${ }^{134}$ D. Stolp,${ }^{134}$ K. Tos, ${ }^{134}$ M. Tripathi, ${ }^{134}$ Z. Wang, ${ }^{134}$ M. Bachtis, ${ }^{135}$ C. Bravo, ${ }^{135}$ R. Cousins, ${ }^{135}$ A. Dasgupta, ${ }^{135}$ A. Florent, ${ }^{135}$ J. Hauser, ${ }^{135}$ M. Ignatenko, ${ }^{135}$ N. Mccoll, ${ }^{135}$ D. Saltzberg, ${ }^{135}$ C. Schnaible, ${ }^{135}$ V. Valuev, ${ }^{135}$ E. Bouvier,${ }^{136}$ K. Burt, ${ }^{136}$ R. Clare,,${ }^{136}$ J. Ellison, ${ }^{136}$ J. W. Gary, ${ }^{136}$ S. M. A. Ghiasi Shirazi, ${ }^{136}$ G. Hanson, ${ }^{136}$ J. Heilman, ${ }^{136}$ P. Jandir, ${ }^{136}$ E. Kennedy, ${ }^{136}$ F. Lacroix, ${ }^{136}$ O. R. Long, ${ }^{136}$ M. Olmedo Negrete, ${ }^{136}$ M. I. Paneva, ${ }^{136}$ A. Shrinivas, ${ }^{136}$ W. Si, ${ }^{136}$ L. Wang, ${ }^{136}$ H. Wei, ${ }^{136}$ S. Wimpenny, ${ }^{136}$ B. R. Yates ${ }^{136}$ J. G. Branson, ${ }^{137}$ S. Cittolin,${ }^{137}$ M. Derdzinski, ${ }^{137}$ R. Gerosa,${ }^{137}$ B. Hashemi, ${ }^{137}$ A. Holzner, ${ }^{137}$ D. Klein, ${ }^{137}$ G. Kole,${ }^{137}$ V. Krutelyov, ${ }^{137}$ J. Letts, ${ }^{137}$ I. Macneill, ${ }^{137}$ M. Masciovecchio, ${ }^{137}$ D. Olivito, ${ }^{137}$ S. Padhi, ${ }^{137}$ M. Pieri, ${ }^{137}$ M. Sani, ${ }^{137}$ V. Sharma, ${ }^{137}$ S. Simon, ${ }^{137}$ M. Tadel, ${ }^{137}$ A. Vartak, ${ }^{137}$ S. Wasserbaech, ${ }^{137, n n n}$ J. Wood, ${ }^{137}$ F. Würthwein, ${ }^{137}$ A. Yagil, ${ }^{137}$ G. Zevi Della Porta ${ }^{137}$ N. Amin, ${ }^{138}$ R. Bhandari, ${ }^{138}$ J. Bradmiller-Feld, ${ }^{138}$ C. Campagnari, ${ }^{138}$ A. Dishaw, ${ }^{138}$ V. Dutta, ${ }^{138}$ M. Franco Sevilla, ${ }^{138}$ C. George, ${ }^{138}$ F. Golf, ${ }^{138}$ L. Gouskos, ${ }^{138}$ J. Gran, ${ }^{138}$ R. Heller ${ }^{138}$ J. Incandela, ${ }^{138}$ S. D. Mullin, ${ }^{138}$ A. Ovcharova, ${ }^{138}$ H. Qu, ${ }^{138}$ J. Richman, ${ }^{138}$ D. Stuart, ${ }^{138}$ I. Suarez, ${ }^{138}$ J. Yoo, ${ }^{138}$ D. Anderson, ${ }^{139}$ J. Bendavid ${ }^{139}$ A. Bornheim, ${ }^{139}$ J. M. Lawhorn, ${ }^{139}$ H. B. Newman, ${ }^{139}$ T. Nguyen, ${ }^{139}$ C. Pena, ${ }^{139}$ M. Spiropulu, ${ }^{139}$ J. R. Vlimant, ${ }^{139}$ S. Xie, ${ }^{139}$ Z. Zhang, ${ }^{139}$ R. Y. Zhu, ${ }^{139}$ M. B. Andrews, ${ }^{140}$ T. Ferguson, ${ }^{140}$ T. Mudholkar, ${ }^{140}$ M. Paulini, ${ }^{140}$ J. Russ, ${ }^{140}$ M. Sun, ${ }^{140}$ H. Vogel, ${ }^{140}$ I. Vorobiev, ${ }^{140}$ M. Weinberg, ${ }^{140}$ J. P. Cumalat, ${ }^{141}$ W. T. Ford,${ }^{141}$ F. Jensen, ${ }^{141}$ A. Johnson, ${ }^{141}$ M. Krohn, ${ }^{141}$ S. Leontsinis, ${ }^{141}$ T. Mulholland, ${ }^{141}$ K. Stenson, ${ }^{141}$ S. R. Wagner, ${ }^{141}$ J. Alexander, ${ }^{142}$ J. Chaves, ${ }^{142}$ J. Chu, ${ }^{142}$ S. Dittmer, ${ }^{142}$ K. Mcdermott, ${ }^{142}$ N. Mirman, ${ }^{142}$ J. R. Patterson, ${ }^{142}$ A. Rinkevicius, ${ }^{142}$ A. Ryd,${ }^{142}$ L. Skinnari, ${ }^{142}$ L. Soffi, ${ }^{142}$ S. M. Tan,${ }^{142}$ Z. Tao, ${ }^{142}$ J. Thom, ${ }^{142}$ J. Tucker, ${ }^{142}$ P. Wittich, ${ }^{142}$ M. Zientek,${ }^{142}$ S. Abdullin, ${ }^{143}$ M. Albrow ${ }^{143}$ G. Apollinari, ${ }^{143}$ A. Apresyan, ${ }^{143}$ A. Apyan, ${ }^{143}$ S. Banerjee, ${ }^{143}$ L. A. T. Bauerdick, ${ }^{143}$ A. Beretvas, ${ }^{143}$ J. Berryhill, ${ }^{143}$ P. C. Bhat, ${ }^{143}$ G. Bolla, ${ }^{143}$ K. Burkett, ${ }^{143}$ J. N. Butler, ${ }^{143}$ A. Canepa, ${ }^{143}$ G. B. Cerati, ${ }^{143}$ H. W. K. Cheung, ${ }^{143}$ F. Chlebana, ${ }^{143}$ M. Cremonesi, ${ }^{143}$ J. Duarte, ${ }^{143}$ V. D. Elvira, ${ }^{143}$ J. Freeman, ${ }^{143}$ Z. Gecse, ${ }^{143}$ E. Gottschalk, ${ }^{143}$ L. Gray, ${ }^{143}$ D. Green, ${ }^{143}$ S. Grünendahl, ${ }^{143}$ O. Gutsche, ${ }^{143}$ R. M. Harris, ${ }^{143}$ S. Hasegawa,${ }^{143}$ J. Hirschauer,${ }^{143}$ Z. Hu, ${ }^{143}$ B. Jayatilaka, ${ }^{143}$ S. Jindariani, ${ }^{143}$ M. Johnson, ${ }^{143}$ U. Joshi, ${ }^{143}$ B. Klima, ${ }^{143}$ B. Kreis, ${ }^{143}$ S. Lammel, ${ }^{143}$ D. Lincoln, ${ }^{143}$ R. Lipton, ${ }^{143}$ M. Liu, ${ }^{143}$ T. Liu, ${ }^{143}$ R. Lopes De Sá, ${ }^{143}$ J. Lykken, ${ }^{143}$ K. Maeshima, ${ }^{143}$ N. Magini, ${ }^{143}$ J. M. Marraffino, ${ }^{143}$ S. Maruyama, ${ }^{143}$ D. Mason, ${ }^{143}$ P. McBride,${ }^{143}$ P. Merkel,${ }^{143}$ S. Mrenna, ${ }^{143}$ S. Nahn, ${ }^{143}$ V. O’Dell, ${ }^{143}$ K. Pedro, ${ }^{143}$ O. Prokofyev, ${ }^{143}$ G. Rakness, ${ }^{143}$ L. Ristori, ${ }^{143}$ B. Schneider, ${ }^{143}$ E. Sexton-Kennedy, ${ }^{143}$ A. Soha, ${ }^{143}$ W. J. Spalding, ${ }^{143}$ L. Spiegel, ${ }^{143}$ S. Stoynev, ${ }^{143}$ J. Strait, ${ }^{143}$ N. Strobbe,${ }^{143}$ L. Taylor, ${ }^{143}$ S. Tkaczyk, ${ }^{143}$ N. V. Tran, ${ }^{143}$ L. Uplegger, ${ }^{143}$ E. W. Vaandering, ${ }^{143}$ C. Vernieri, ${ }^{143}$ M. Verzocchi, ${ }^{143}$ R. Vidal,${ }^{143}$ M. Wang, ${ }^{143}$ H. A. Weber,${ }^{143}$ A. Whitbeck,${ }^{143}$ D. Acosta, ${ }^{144}$ P. Avery, ${ }^{144}$ P. Bortignon, ${ }^{144}$ D. Bourilkov, ${ }^{144}$ A. Brinkerhoff, ${ }^{144}$ A. Carnes, ${ }^{144}$ M. Carver, ${ }^{144}$ D. Curry, ${ }^{144}$ R. D. Field,${ }^{144}$ I. K. Furic, ${ }^{144}$ J. Konigsberg, ${ }^{144}$ A. Korytov, ${ }^{144}$ K. Kotov, ${ }^{144}$ P. Ma ${ }^{144}$ K. Matchev, ${ }^{144}$ H. Mei,${ }^{144}$ G. Mitselmakher, ${ }^{144}$ D. Rank, ${ }^{144}$ D. Sperka ${ }^{144}$ N. Terentyev, ${ }^{144}$ L. Thomas, ${ }^{144}$ J. Wang, ${ }^{144}$ S. Wang, ${ }^{144}$ J. Yelton, ${ }^{144}$ Y. R. Joshi, ${ }^{145}$ S. Linn, ${ }^{145}$ P. Markowitz, ${ }^{145}$ J. L. Rodriguez, ${ }^{145}$ A. Ackert, ${ }^{146}$ T. Adams, ${ }^{146}$ A. Askew, ${ }^{146}$ S. Hagopian, ${ }^{146}$ V. Hagopian, ${ }^{146}$ K. F. Johnson, ${ }^{146}$ T. Kolberg, ${ }^{146}$ G. Martinez, ${ }^{146}$ T. Perry, ${ }^{146}$ H. Prosper, ${ }^{146}$ A. Saha, ${ }^{146}$ A. Santra, ${ }^{146}$ R. Yohay, ${ }^{146}$ M. M. Baarmand, ${ }^{147}$ V. Bhopatkar, ${ }^{147}$ S. Colafranceschi, ${ }^{147}$ M. Hohlmann, ${ }^{147}$ D. Noonan, ${ }^{147}$ T. Roy ${ }^{147}$ F. Yumiceva, ${ }^{147}$ M. R. Adams, ${ }^{148}$ L. Apanasevich, ${ }^{148}$ D. Berry, ${ }^{148}$ R. R. Betts, ${ }^{148}$ R. Cavanaugh, ${ }^{148}$ X. Chen, ${ }^{148}$ O. Evdokimov, ${ }^{148}$ C. E. Gerber, ${ }^{148}$ D. A. Hangal, ${ }^{148}$ D. J. Hofman, ${ }^{148}$ K. Jung, ${ }^{148}$ J. Kamin, ${ }^{148}$ I. D. Sandoval Gonzalez, ${ }^{148}$ M. B. Tonjes, ${ }^{148}$ H. Trauger, ${ }^{148}$ N. Varelas, ${ }^{148}$ H. Wang, ${ }^{148}$ Z. Wu, ${ }^{148}$ J. Zhang, ${ }^{148}$ B. Bilki, ${ }^{149,000}$ W. Clarida, ${ }^{149}$ K. Dilsiz, ${ }^{149, p p p}$ S. Durgut, ${ }^{149}$ R. P. Gandrajula, ${ }^{149}$ M. Haytmyradov, ${ }^{149}$ V. Khristenko, ${ }^{149}$ J.-P. Merlo, ${ }^{149}$ H. Mermerkaya, ${ }^{149, q q 9}$ A. Mestvirishvili, ${ }^{149}$ A. Moeller, ${ }^{149}$ J. Nachtman, ${ }^{149}$ H. Ogul, ${ }^{149, \text { rrr }}$ Y. Onel, ${ }^{149}$ F. Ozok, ${ }^{149, \text { ss }}$ A. Penzo, ${ }^{149}$ C. Snyder, ${ }^{149}$ E. Tiras, ${ }^{149}$ J. Wetzel, ${ }^{149}$ K. Yi, ${ }^{149}$ B. Blumenfeld, ${ }^{150}$ A. Cocoros, ${ }^{150}$ N. Eminizer, ${ }^{150}$ D. Fehling, ${ }^{150}$ L. Feng, ${ }^{150}$ A. V. Gritsan, ${ }^{150}$ P. Maksimovic, ${ }^{150}$ J. Roskes, ${ }^{150}$ U. Sarica, ${ }^{150}$ M. Swartz, ${ }^{150}$ M. Xiao, ${ }^{150}$ C. You, ${ }^{150}$ A. Al-bataineh, ${ }^{151}$ P. Baringer, ${ }^{151}$ A. Bean, ${ }^{151}$ S. Boren, ${ }^{151}$ J. Bowen, ${ }^{151}$ J. Castle, ${ }^{151}$ S. Khali1, ${ }^{151}$ A. Kropivnitskaya, ${ }^{151}$ D. Majumder,${ }^{151}$ W. Mcbrayer,${ }^{151}$ M. Murray,${ }^{151}$ C. Royon, ${ }^{151}$ S. Sanders, ${ }^{151}$ E. Schmitz, ${ }^{151}$ R. Stringer, ${ }^{151}$ J. D. Tapia Takaki, ${ }^{151}$ Q. Wang, ${ }^{151}$ A. Ivanov, ${ }^{152}$ K. Kaadze, ${ }^{152}$ Y. Maravin, ${ }^{152}$ A. Mohammadi, ${ }^{152}$ L. K. Saini, ${ }^{152}$ N. Skhirtladze, ${ }^{152}$ S. Toda,${ }^{152}$ F. Rebassoo, ${ }^{153}$ D. Wright, ${ }^{153}$ C. Anelli, ${ }^{154}$ A. Baden, ${ }^{154}$ 
O. Baron, ${ }^{154}$ A. Belloni, ${ }^{154}$ B. Calvert, ${ }^{154}$ S. C. Eno, ${ }^{154}$ C. Ferraioli, ${ }^{154}$ N. J. Hadley, ${ }^{154}$ S. Jabeen, ${ }^{154}$ G. Y. Jeng, ${ }^{154}$ R. G. Kellogg, ${ }^{154}$ J. Kunkle, ${ }^{154}$ A. C. Mignerey, ${ }^{154}$ F. Ricci-Tam, ${ }^{154}$ Y. H. Shin,${ }^{154}$ A. Skuja, ${ }^{154}$ S. C. Tonwar, ${ }^{154}$ D. Abercrombie, ${ }^{155}$ B. Allen, ${ }^{155}$ V. Azzolini, ${ }^{155}$ R. Barbieri, ${ }^{155}$ A. Baty, ${ }^{155}$ R. Bi,${ }^{155}$ S. Brandt, ${ }^{155}$ W. Busza, ${ }^{155}$ I. A. Cali, ${ }^{155}$ M. D’Alfonso, ${ }^{155}$ Z. Demiragli, ${ }^{155}$ G. Gomez Ceballos, ${ }^{155}$ M. Goncharov, ${ }^{155}$ D. Hsu, ${ }^{155}$ Y. Iiyama, ${ }^{155}$ G. M. Innocenti, ${ }^{155}$ M. Klute, ${ }^{155}$ D. Kovalskyi, ${ }^{155}$ Y. S. Lai, ${ }^{155}$ Y.-J. Lee, ${ }^{155}$ A. Levin, ${ }^{155}$ P. D. Luckey, ${ }^{155}$ B. Maier, ${ }^{155}$ A. C. Marini, ${ }^{155}$ C. Mcginn, ${ }^{155}$ C. Mironov, ${ }^{155}$ S. Narayanan, ${ }^{155}$ X. Niu, ${ }^{155}$ C. Paus, ${ }^{155}$ C. Roland,${ }^{155}$ G. Roland, ${ }^{155}$ J. Salfeld-Nebgen, ${ }^{155}$ G. S. F. Stephans, ${ }^{155}$ K. Tatar ${ }^{155}$ D. Velicanu, ${ }^{155}$ J. Wang, ${ }^{155}$ T. W. Wang, ${ }^{155}$ B. Wyslouch, ${ }^{155}$ A. C. Benvenuti, ${ }^{156}$ R. M. Chatterjee, ${ }^{156}$ A. Evans, ${ }^{156}$ P. Hansen, ${ }^{156}$ S. Kalafut, ${ }^{156}$ Y. Kubota, ${ }^{156}$ Z. Lesko, ${ }^{156}$ J. Mans, ${ }^{156}$ S. Nourbakhsh, ${ }^{156}$ N. Ruckstuhl, ${ }^{156}$ R. Rusack, ${ }^{156}$ J. Turkewitz, ${ }^{156}$ J. G. Acosta, ${ }^{157}$ S. Oliveros, ${ }^{157}$ E. Avdeeva, ${ }^{158}$ K. Bloom, ${ }^{158}$ D. R. Claes, ${ }^{158}$ C. Fangmeier, ${ }^{158}$ R. Gonzalez Suarez, ${ }^{158}$ R. Kamalieddin, ${ }^{158}$ I. Kravchenko, ${ }^{158}$ J. Monroy, ${ }^{158}$ J. E. Siado, ${ }^{158}$ G. R. Snow, ${ }^{158}$ B. Stieger, ${ }^{158}$ M. Alyari, ${ }^{159}$ J. Dolen, ${ }^{159}$ A. Godshalk, ${ }^{159}$ C. Harrington, ${ }^{159}$ I. Iashvili, ${ }^{159}$ D. Nguyen, ${ }^{159}$ A. Parker, ${ }^{159}$ S. Rappoccio, ${ }^{159}$ B. Roozbahani, ${ }^{159}$ G. Alverson, ${ }^{160}$ E. Barberis, ${ }^{160}$ A. Hortiangtham, ${ }^{160}$ A. Massironi, ${ }^{160}$ D. M. Morse, ${ }^{160}$ D. Nash ${ }^{160}$ T. Orimoto, ${ }^{160}$ R. Teixeira De Lima ${ }^{160}$ D. Trocino, ${ }^{160}$ D. Wood, ${ }^{160}$ S. Bhattacharya, ${ }^{161}$ O. Charaf, ${ }^{161}$ K. A. Hahn, ${ }^{161}$ N. Mucia, ${ }^{161}$ N. Odell, ${ }^{161}$ B. Pollack, ${ }^{161}$ M. H. Schmitt, ${ }^{161}$ K. Sung, ${ }^{161}$ M. Trovato, ${ }^{161}$ M. Velasco, ${ }^{161}$ N. Dev, ${ }^{162}$ M. Hildreth, ${ }^{162}$ K. Hurtado Anampa ${ }^{162}$ C. Jessop, ${ }^{162}$ D. J. Karmgard, ${ }^{162}$ N. Kellams, ${ }^{162}$ K. Lannon, ${ }^{162}$ N. Loukas, ${ }^{162}$ N. Marinelli, ${ }^{162}$ F. Meng, ${ }^{162}$ C. Mueller, ${ }^{162}$ Y. Musienko, ${ }^{162, k \mathrm{k}}$ M. Planer, ${ }^{162}$ A. Reinsvold, ${ }^{162}$ R. Ruchti, ${ }^{162}$ G. Smith, ${ }^{162}$ S. Taroni, ${ }^{162}$ M. Wayne, ${ }^{162}$ M. Wolf, ${ }^{162}$ A. Woodard,${ }^{162}$ J. Alimena, ${ }^{163}$ L. Antonelli, ${ }^{163}$ B. Bylsma, ${ }^{163}$ L. S. Durkin, ${ }^{163}$ S. Flowers ${ }^{163}$ B. Francis, ${ }^{163}$ A. Hart, ${ }^{163}$ C. Hill, ${ }^{163}$ W. Ji, ${ }^{163}$ B. Liu, ${ }^{163}$ W. Luo, ${ }^{163}$ D. Puigh,,${ }^{163}$ B. L. Winer, ${ }^{163}$ H. W. Wulsin, ${ }^{163}$ A. Benaglia, ${ }^{164}$ S. Cooperstein, ${ }^{164}$ O. Driga,${ }^{164}$ P. Elmer, ${ }^{164}$ J. Hardenbrook, ${ }^{164}$ P. Hebda, ${ }^{164}$ S. Higginbotham, ${ }^{164}$ D. Lange, ${ }^{164}$ J. Luo, ${ }^{164}$ D. Marlow, ${ }^{164}$ K. Mei ${ }^{164}$ I. Ojalvo, ${ }^{164}$ J. Olsen, ${ }^{164}$ C. Palmer, ${ }^{164}$ P. Piroué, ${ }^{164}$ D. Stickland ${ }^{164}$ C. Tully, ${ }^{164}$ S. Malik, ${ }^{165}$ S. Norberg, ${ }^{165}$ A. Barker, ${ }^{166}$ V. E. Barnes, ${ }^{166}$ S. Das, ${ }^{166}$ S. Folgueras, ${ }^{166}$ L. Gutay, ${ }^{166}$ M. K. Jha ${ }^{166}$ M. Jones, ${ }^{166}$ A. W. Jung, ${ }^{166}$ A. Khatiwada, ${ }^{166}$ D. H. Miller, ${ }^{166}$ N. Neumeister,${ }^{166}$ C. C. Peng, ${ }^{166}$ J. F. Schulte, ${ }^{166}$ J. Sun, ${ }^{166}$ F. Wang, ${ }^{166}$ W. Xie, ${ }^{166}$ T. Cheng, ${ }^{167}$ N. Parashar, ${ }^{167}$ J. Stupak, ${ }^{167}$ A. Adair, ${ }^{168}$ B. Akgun, ${ }^{168}$ Z. Chen, ${ }^{168}$ K. M. Ecklund, ${ }^{168}$ F. J. M. Geurts, ${ }^{168}$ M. Guilbaud, ${ }^{168}$ W. Li, ${ }^{168}$ B. Michlin, ${ }^{168}$ M. Northup,${ }^{168}$ B. P. Padley,${ }^{168}$ J. Roberts, ${ }^{168}$ J. Rorie, ${ }^{168}$ Z. Tu, ${ }^{168}$ J. Zabel, ${ }^{168}$ A. Bodek, ${ }^{169}$ P. de Barbaro, ${ }^{169}$ R. Demina, ${ }^{169}$ Y. t. Duh, ${ }^{169}$ T. Ferbel, ${ }^{169}$ M. Galanti, ${ }^{169}$ A. Garcia-Bellido, ${ }^{169}$ J. Han, ${ }^{169}$ O. Hindrichs, ${ }^{169}$ A. Khukhunaishvili, ${ }^{169}$ K. H. Lo, ${ }^{169}$ P. Tan, ${ }^{169}$ M. Verzetti, ${ }^{169}$ R. Ciesielski, ${ }^{170}$ K. Goulianos, ${ }^{170}$ C. Mesropian, ${ }^{170}$ A. Agapitos, ${ }^{171}$ J. P. Chou, ${ }^{171}$ M. Christos,${ }^{171}$ K. Feigelis, ${ }^{171}$ Y. Gershtein, ${ }^{171}$ T. A. Gómez Espinosa, ${ }^{171}$ E. Halkiadakis, ${ }^{171}$ M. Heindl, ${ }^{171}$ E. Hughes, ${ }^{171}$ S. Kaplan, ${ }^{171}$

R. Kunnawalkam Elayavalli, ${ }^{171}$ S. Kyriacou, ${ }^{171}$ A. Lath, ${ }^{171}$ R. Montalvo, ${ }^{171}$ K. Nash, ${ }^{171}$ M. Osherson, ${ }^{171}$ H. Saka, ${ }^{171}$ S. Salur, ${ }^{171}$ S. Schnetzer, ${ }^{171}$ D. Sheffield, ${ }^{171}$ S. Somalwar, ${ }^{171}$ R. Stone, ${ }^{171}$ S. Thomas, ${ }^{171}$ P. Thomassen,${ }^{171}$ M. Walker, ${ }^{171}$ B. Zhou, ${ }^{171}$ A. G. Delannoy, ${ }^{172}$ M. Foerster, ${ }^{172}$ J. Heideman, ${ }^{172}$ G. Riley, ${ }^{172}$ K. Rose,${ }^{172}$ S. Spanier,${ }^{172}$ K. Thapa, ${ }^{172}$ O. Bouhali, ${ }^{173, \text { ttt }}$ A. Castaneda Hernandez, ${ }^{173, t t t}$ A. Celik, ${ }^{173}$ M. Dalchenko, ${ }^{173}$ M. De Mattia, ${ }^{173}$ A. Delgado, ${ }^{173}$ S. Dildick, ${ }^{173}$ R. Eusebi, ${ }^{173}$ J. Gilmore, ${ }^{173}$ T. Huang, ${ }^{173}$ T. Kamon, ${ }^{173, \text { uuu }}$ R. Mueller, ${ }^{173}$ Y. Pakhotin, ${ }^{173}$ R. Patel, ${ }^{173}$ A. Perloff, ${ }^{173}$ L. Perniè, ${ }^{173}$ D. Rathjens, ${ }^{173}$ A. Safonov, ${ }^{173}$ A. Tatarinov, ${ }^{173}$ K. A. Ulmer, ${ }^{173}$ N. Akchurin, ${ }^{174}$ J. Damgov, ${ }^{174}$ F. De Guio, ${ }^{174}$ P. R. Dudero, ${ }^{174}$ J. Faulkner, ${ }^{174}$ E. Gurpinar, ${ }^{174}$ S. Kunori, ${ }^{174}$ K. Lamichhane, ${ }^{174}$ S. W. Lee, ${ }^{174}$ T. Libeiro, ${ }^{174}$ T. Peltola, ${ }^{174}$ S. Undleeb, ${ }^{174}$ I. Volobouev, ${ }^{174}$ Z. Wang, ${ }^{174}$ S. Greene, ${ }^{175}$ A. Gurrola, ${ }^{175}$ R. Janjam, ${ }^{175}$ W. Johns, ${ }^{175}$ C. Maguire, ${ }^{175}$ A. Melo, ${ }^{175} \mathrm{H}$. Ni, ${ }^{175}$ P. Sheldon, ${ }^{175}$ S. Tuo, ${ }^{175}$ J. Velkovska, ${ }^{175}$ Q. Xu ${ }^{175}$ M. W. Arenton, ${ }^{176}$ P. Barria, ${ }^{176}$ B. Cox,${ }^{176}$ R. Hirosky, ${ }^{176}$ A. Ledovskoy, ${ }^{176}$ H. Li ${ }^{176}$ C. Neu, ${ }^{176}$ T. Sinthuprasith, ${ }^{176}$ X. Sun, ${ }^{176}$ Y. Wang, ${ }^{176}$ E. Wolfe, ${ }^{176}$ F. Xia, ${ }^{176}$ R. Harr, ${ }^{177}$ P. E. Karchin,,${ }^{177}$ J. Sturdy, ${ }^{177}$ S. Zaleski, ${ }^{177}$ M. Brodski, ${ }^{178}$ J. Buchanan, ${ }^{178}$ C. Caillol, ${ }^{178}$ S. Dasu, ${ }^{178}$ L. Dodd, ${ }^{178}$ S. Duric, ${ }^{178}$ B. Gomber, ${ }^{178}$ M. Grothe, ${ }^{178}$ M. Herndon, ${ }^{178}$ A. Hervé, ${ }^{178}$ U. Hussain, ${ }^{178}$ P. Klabbers, ${ }^{178}$ A. Lanaro, ${ }^{178}$ A. Levine, ${ }^{178}$ K. Long, ${ }^{178}$ R. Loveless, ${ }^{178}$ G. A. Pierro, ${ }^{178}$ G. Polese, ${ }^{178}$ T. Ruggles, ${ }^{178}$ A. Savin, ${ }^{178}$ N. Smith, ${ }^{178}$ W. H. Smith, ${ }^{178}$ D. Taylor, ${ }^{178}$ and N. Woods ${ }^{178}$

(CMS Collaboration)

\footnotetext{
${ }^{1}$ Yerevan Physics Institute, Yerevan, Armenia

${ }^{2}$ Institut für Hochenergiephysik, Wien, Austria

${ }^{3}$ Institute for Nuclear Problems, Minsk, Belarus

${ }^{4}$ Universiteit Antwerpen, Antwerpen, Belgium
} 


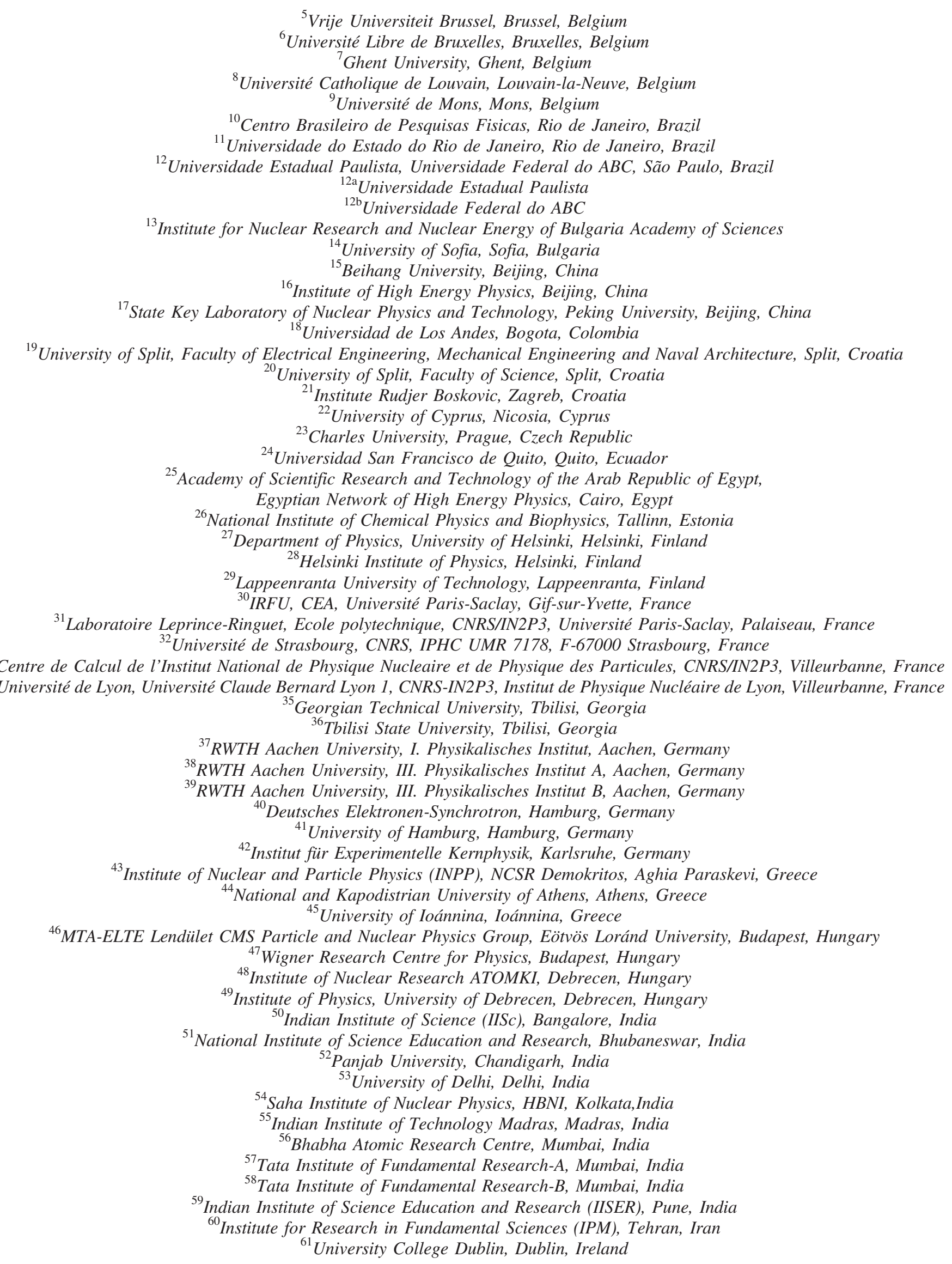


${ }^{62}$ INFN Sezione di Bari, Università di Bari, Politecnico di Bari, Bari, Italy

${ }^{62 \mathrm{a}}$ INFN Sezione di Bari

${ }^{62 \mathrm{~b}}$ Università di Bari

${ }^{62 \mathrm{c}}$ Politecnico di Bari

${ }^{63}$ INFN Sezione di Bologna, Università di Bologna, Bologna, Italy

${ }^{63 a}$ INFN Sezione di Bologna

${ }^{63 \mathrm{~b}}$ Università di Bologna

${ }^{64}$ INFN Sezione di Catania, Università di Catania, Catania, Italy

${ }^{64 a}$ INFN Sezione di Catania

${ }^{64 \mathrm{~b}}$ Università di Catania

${ }^{65}$ INFN Sezione di Firenze, Università di Firenze, Firenze, Italy

${ }^{65}$ aNFN Sezione di Firenze

${ }^{65 \mathrm{~b}}$ Università di Firenze

${ }^{66}$ INFN Laboratori Nazionali di Frascati, Frascati, Italy

${ }^{67}$ INFN Sezione di Genova, Università di Genova, Genova, Italy

${ }^{67 a}$ INFN Sezione di Genova

${ }^{67 b}$ Università di Genova

${ }^{68 \mathrm{a}}$ INFN Sezione di Milano-Bicocca

${ }^{68 \mathrm{~b}}$ Università di Milano-Bicocca

${ }^{69}$ INFN Sezione di Napoli, Università di Napoli 'Federico II', Napoli, Italy, Università della Basilicata,

Potenza, Italy, Università G. Marconi, Roma, Italy

${ }^{69 a}$ INFN Sezione di Napoli

${ }^{69 \mathrm{~b}}$ Università di Napoli 'Federico II'

${ }^{69 \mathrm{c}}$ Università della Basilicata

${ }^{69 \mathrm{~d}}$ Università G. Marconi

${ }^{70}$ INFN Sezione di Padova, Università di Padova, Padova, Italy, Università di Trento, Trento, Italy

${ }^{70 a}$ INFN Sezione di Padova

${ }^{70 \mathrm{~b}}$ Università di Padova

${ }^{70 \mathrm{c}}$ Università di Trento

${ }^{71}$ INFN Sezione di Pavia, Università di Pavia, Pavia, Italy

${ }^{71 a}$ INFN Sezione di Pavia

${ }^{71 b}$ Università di Pavia

${ }^{72}$ INFN Sezione di Perugia, Università di Perugia, Perugia, Italy

${ }^{72 \mathrm{a}}$ INFN Sezione di Perugia

${ }^{72 \mathrm{~b}}$ Università di Perugia

${ }^{73}$ INFN Sezione di Pisa, Università di Pisa, Scuola Normale Superiore di Pisa, Pisa, Italy

${ }^{73 a}$ INFN Sezione di Pisa

${ }^{73 b}$ Università di Pisa

${ }^{73 \mathrm{c}}$ Scuola Normale Superiore di Pisa

${ }^{74}$ INFN Sezione di Roma

${ }^{74 \mathrm{~b}}$ Sapienza Università di Roma

${ }^{75}$ INFN Sezione di Torino, Università di Torino, Torino, Italy, Università del Piemonte Orientale, Novara, Italy

${ }^{75 a}$ INFN Sezione di Torino

${ }^{75 b}$ Università di Torino

${ }^{75 \mathrm{c}}$ Università del Piemonte Orientale

${ }^{76}$ INFN Sezione di Trieste, Università di Trieste, Trieste, Italy

${ }^{76 a}$ INFN Sezione di Trieste

${ }^{76 \mathrm{~b}}$ Università di Trieste

${ }^{77}$ Kyungpook National University, Daegu, Korea

${ }^{78}$ Chonbuk National University, Jeonju, Korea

${ }^{79}$ Chonnam National University, Institute for Universe and Elementary Particles, Kwangju, Korea

${ }^{80}$ Hanyang University, Seoul, Korea

${ }^{81}$ Korea University, Seoul, Korea

${ }^{82}$ Seoul National University, Seoul, Korea

${ }^{83}$ University of Seoul, Seoul, Korea

${ }^{84}$ Sungkyunkwan University, Suwon, Korea

${ }^{85}$ Vilnius University, Vilnius, Lithuania

${ }^{86}$ National Centre for Particle Physics, Universiti Malaya, Kuala Lumpur, Malaysia

${ }^{87}$ Centro de Investigacion y de Estudios Avanzados del IPN, Mexico City, Mexico

${ }^{88}$ Universidad Iberoamericana, Mexico City, Mexico 


\footnotetext{
${ }^{89}$ Benemerita Universidad Autonoma de Puebla, Puebla, Mexico

${ }^{90}$ Universidad Autónoma de San Luis Potosí, San Luis Potosí, Mexico

${ }^{91}$ University of Auckland, Auckland, New Zealand

${ }^{92}$ University of Canterbury, Christchurch, New Zealand

${ }^{93}$ National Centre for Physics, Quaid-I-Azam University, Islamabad, Pakistan

${ }^{94}$ National Centre for Nuclear Research, Swierk, Poland

${ }^{95}$ Institute of Experimental Physics, Faculty of Physics, University of Warsaw, Warsaw, Poland

${ }^{96}$ Laboratório de Instrumentação e Física Experimental de Partículas, Lisboa, Portugal

${ }^{97}$ Joint Institute for Nuclear Research, Dubna, Russia

${ }^{98}$ Petersburg Nuclear Physics Institute, Gatchina (St. Petersburg), Russia

${ }^{99}$ Institute for Nuclear Research, Moscow, Russia

${ }^{100}$ Institute for Theoretical and Experimental Physics, Moscow, Russia

${ }^{101}$ Moscow Institute of Physics and Technology, Moscow, Russia

${ }^{102}$ National Research Nuclear University 'Moscow Engineering Physics Institute' (MEPhI), Moscow, Russia

${ }^{103}$ P.N. Lebedev Physical Institute, Moscow, Russia

${ }^{104}$ Skobeltsyn Institute of Nuclear Physics, Lomonosov Moscow State University, Moscow, Russia

${ }^{105}$ Novosibirsk State University (NSU), Novosibirsk, Russia

${ }^{106}$ State Research Center of Russian Federation, Institute for High Energy Physics, Protvino, Russia

${ }^{107}$ University of Belgrade, Faculty of Physics and Vinca Institute of Nuclear Sciences, Belgrade, Serbia

${ }^{108}$ Centro de Investigaciones Energéticas Medioambientales y Tecnológicas (CIEMAT), Madrid, Spain

${ }^{109}$ Universidad Autónoma de Madrid, Madrid, Spain

${ }^{110}$ Universidad de Oviedo, Oviedo, Spain

${ }^{111}$ Instituto de Física de Cantabria (IFCA), CSIC-Universidad de Cantabria, Santander, Spain

${ }^{112}$ CERN, European Organization for Nuclear Research, Geneva, Switzerland

${ }^{113}$ Paul Scherrer Institut, Villigen, Switzerland

${ }^{114}$ Institute for Particle Physics, ETH Zurich, Zurich, Switzerland

${ }^{115}$ Universität Zürich, Zurich, Switzerland

${ }^{116}$ National Central University, Chung-Li, Taiwan

${ }^{117}$ National Taiwan University (NTU), Taipei, Taiwan

${ }^{118}$ Chulalongkorn University, Faculty of Science, Department of Physics, Bangkok, Thailand

${ }^{119}$ Cukurova University, Physics Department, Science and Art Faculty, Adana, Turkey

${ }^{120}$ Middle East Technical University, Physics Department, Ankara, Turkey

${ }^{121}$ Bogazici University, Istanbul, Turkey

${ }^{122}$ Istanbul Technical University, Istanbul, Turkey

${ }^{123}$ Institute for Scintillation Materials of National Academy of Science of Ukraine, Kharkov, Ukraine

${ }^{124}$ National Scientific Center, Kharkov Institute of Physics and Technology, Kharkov, Ukraine

${ }^{125}$ University of Bristol, Bristol, United Kingdom

${ }^{126}$ Rutherford Appleton Laboratory, Didcot, United Kingdom

${ }^{127}$ Imperial College, London, United Kingdom

${ }^{128}$ Brunel University, Uxbridge, United Kingdom

${ }^{129}$ Baylor University, Waco, USA

${ }^{130}$ Catholic University of America, Washington DC, USA

${ }^{131}$ The University of Alabama, Tuscaloosa, USA

${ }^{132}$ Boston University, Boston, USA

${ }^{133}$ Brown University, Providence, USA

${ }^{134}$ University of California, Davis, Davis, USA

${ }^{135}$ University of California, Los Angeles, USA

${ }^{136}$ University of California, Riverside, Riverside, USA

${ }^{137}$ University of California, San Diego, La Jolla, USA

${ }^{138}$ University of California, Santa Barbara-Department of Physics, Santa Barbara, USA

${ }^{139}$ California Institute of Technology, Pasadena, USA

${ }^{140}$ Carnegie Mellon University, Pittsburgh, USA

${ }^{141}$ University of Colorado Boulder, Boulder, USA

${ }^{142}$ Cornell University, Ithaca, USA

${ }^{143}$ Fermi National Accelerator Laboratory, Batavia, USA

${ }^{144}$ University of Florida, Gainesville, USA

${ }^{145}$ Florida International University, Miami, USA

${ }^{146}$ Florida State University, Tallahassee, USA

${ }^{147}$ Florida Institute of Technology, Melbourne, USA

${ }^{148}$ University of Illinois at Chicago (UIC), Chicago, USA
} 


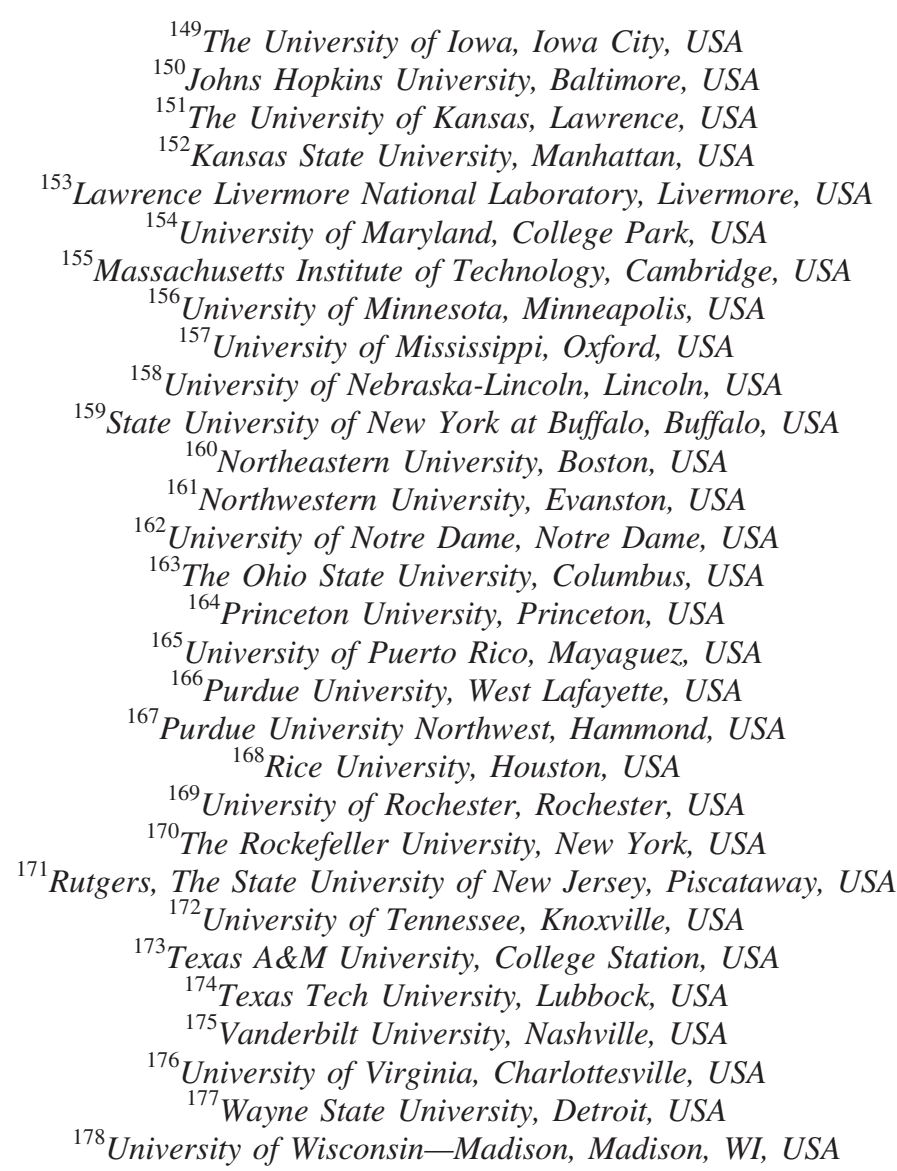

${ }^{\mathrm{a}}$ Deceased.

${ }^{\mathrm{b}}$ Also at Vienna University of Technology, Vienna, Austria

${ }^{\mathrm{c}}$ Also at State Key Laboratory of Nuclear Physics and Technology, Peking University, Beijing, China

${ }^{\mathrm{d}}$ Also at Universidade Estadual de Campinas, Campinas, Brazil

${ }^{\mathrm{e}}$ Also at Universidade Federal de Pelotas, Pelotas, Brazil

${ }^{\mathrm{f}}$ Also at Université Libre de Bruxelles, Bruxelles, Belgium

${ }^{\mathrm{g}}$ Also at Institute for Theoretical and Experimental Physics, Moscow, Russia

${ }^{\mathrm{h}}$ Also at Joint Institute for Nuclear Research, Dubna, Russia

${ }^{1}$ Also at Ain Shams University, Cairo, Egypt

${ }^{\mathrm{j}}$ Also at British University in Egypt, Cairo, Egypt

${ }^{\mathrm{k}}$ Also at Cairo University, Cairo, Egypt

${ }^{1}$ Also at Université de Haute Alsace, Mulhouse, France

${ }^{m}$ Also at Skobeltsyn Institute of Nuclear Physics, Lomonosov Moscow State University, Moscow, Russia

${ }^{\mathrm{n}}$ Also at Tbilisi State University, Tbilisi, Georgia

${ }^{\circ}$ Also at CERN, European Organization for Nuclear Research, Geneva, Switzerland

${ }^{\mathrm{p}}$ Also at RWTH Aachen University, III. Physikalisches Institut A, Aachen, Germany

${ }^{\mathrm{q}}$ Also at University of Hamburg, Hamburg, Germany

${ }^{\mathrm{r}}$ Also at Brandenburg University of Technology, Cottbus, Germany

${ }^{\mathrm{s}}$ Also at Institute of Nuclear Research ATOMKI, Debrecen, Hungary

${ }^{t}$ Also at MTA-ELTE Lendület CMS Particle and Nuclear Physics Group, Eötvös Loránd University, Budapest, Hungary

${ }^{u}$ Also at Institute of Physics, University of Debrecen, Debrecen, Hungary

${ }^{v}$ Also at IIT Bhubaneswar, Bhubaneswar, India

${ }^{\mathrm{w}}$ Also at Institute of Physics, Bhubaneswar, India

${ }^{\mathrm{x}}$ Also at University of Visva-Bharati, Santiniketan, India

${ }^{\mathrm{y}}$ Also at University of Ruhuna, Matara, Sri Lanka

${ }^{\mathrm{z}}$ Also at Isfahan University of Technology, Isfahan, Iran

${ }^{\text {aa }}$ Also at Yazd University, Yazd, Iran

${ }^{\mathrm{bb}}$ Also at Plasma Physics Research Center, Science and Research Branch, Islamic Azad University, Tehran, Iran

${ }^{\mathrm{cc}}$ Also at Università degli Studi di Siena, Siena, Italy 
${ }^{\text {dd }}$ Also at INFN Sezione di Milano-Bicocca, Università di Milano-Bicocca, Milano, Italy

${ }^{\mathrm{ee}}$ Also at Purdue University, West Lafayette, USA

${ }^{\mathrm{ff}}$ Also at International Islamic University of Malaysia, Kuala Lumpur, Malaysia

${ }^{\mathrm{gg}}$ Also at Malaysian Nuclear Agency, MOSTI, Kajang, Malaysia

${ }^{\text {hh }}$ Also at Consejo Nacional de Ciencia y Tecnología, Mexico city, Mexico

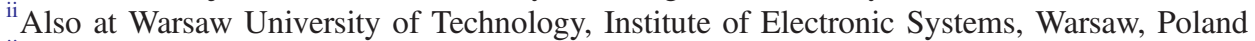

${ }^{\mathrm{jj}}$ Also at Czech Technical University, Praha, Czech Republic

${ }^{\mathrm{kk}}$ Also at Institute for Nuclear Research, Moscow, Russia

"Ilso at National Research Nuclear University 'Moscow Engineering Physics Institute' (MEPhI), Moscow, Russia

${ }^{\mathrm{mm}}$ Also at St. Petersburg State Polytechnical University, St. Petersburg, Russia

${ }^{\mathrm{nn}}$ Also at University of Florida, Gainesville, USA

${ }^{\circ 0}$ Also at P.N. Lebedev Physical Institute, Moscow, Russia

${ }^{p p}$ Also at California Institute of Technology, Pasadena, USA

${ }^{\mathrm{qq}}$ Also at Budker Institute of Nuclear Physics, Novosibirsk, Russia

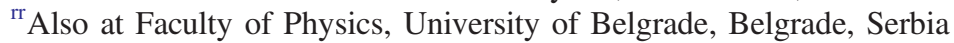

${ }^{\text {ss }}$ Also at INFN Sezione di Roma, Sapienza Università di Roma, Rome, Italy

${ }^{\text {th} A l s o ~ a t ~ U n i v e r s i t y ~ o f ~ B e l g r a d e, ~ F a c u l t y ~ o f ~ P h y s i c s ~ a n d ~ V i n c a ~ I n s t i t u t e ~ o f ~ N u c l e a r ~ S c i e n c e s, ~ B e l g r a d e, ~ S e r b i a ~}$

${ }^{u u}$ Also at Scuola Normale e Sezione dell'INFN, Pisa, Italy

${ }^{\mathrm{vv}}$ Also at National and Kapodistrian University of Athens, Athens, Greece

${ }^{w w}$ Also at Riga Technical University, Riga, Latvia

${ }^{\mathrm{xx}}$ Also at Universität Zürich, Zurich, Switzerland

${ }^{y y}$ Also at Stefan Meyer Institute for Subatomic Physics

${ }^{\mathrm{zz}}$ Also at Istanbul University, Faculty of Science, Istanbul, Turkey

${ }^{a a a}$ Also at Adiyaman University, Adiyaman, Turkey

${ }^{\mathrm{bbb}}$ Also at Istanbul Aydin University, Istanbul, Turkey

${ }^{c c c}$ Also at Mersin University, Mersin, Turkey

${ }^{\text {ddd }}$ Also at Cag University, Mersin, Turkey

${ }^{e e e}$ Also at Piri Reis University, Istanbul, Turkey

${ }^{\text {fff }}$ Also at Izmir Institute of Technology, Izmir, Turkey

${ }^{\text {ggg }}$ Also at Necmettin Erbakan University, Konya, Turkey

${ }^{\text {hhh }}$ Also at Marmara University, Istanbul, Turkey

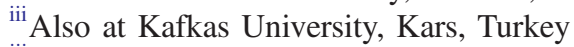

${ }^{\mathrm{ijj}}$ Also at Istanbul Bilgi University, Istanbul, Turkey

${ }^{k k k}$ Also at Rutherford Appleton Laboratory, Didcot, United Kingdom

${ }^{111}$ Also at School of Physics and Astronomy, University of Southampton, Southampton, United Kingdom

${ }^{\mathrm{mmm}}$ Also at Instituto de Astrofísica de Canarias, La Laguna, Spain

${ }^{\mathrm{nnn}}$ Also at Utah Valley University, Orem, USA

${ }^{000}$ Also at Beykent University

${ }^{\mathrm{ppp}}$ Also at Bingol University, Bingol, Turkey

${ }^{\mathrm{qqq}}$ Also at Erzincan University, Erzincan, Turkey

${ }^{\text {rrr }}$ Also at Sinop University, Sinop, Turkey

${ }^{\text {sss }}$ Also at Mimar Sinan University, Istanbul, Istanbul, Turkey

ttt Also at Texas A\&M University at Qatar, Doha, Qatar

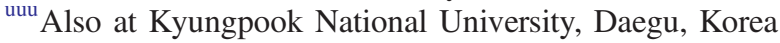

